\title{
Constructing Discrete Chaotic Systems with Positive Lyapunov Exponents
}

\author{
Chuanfu Wang*, Chunlei Fan ${ }^{\dagger}$ and Qun Ding ${ }^{\ddagger}$ \\ Electronic Engineering College, Heilongjiang University, \\ Harbin 150080, P. R. China \\ *chuanfuwang@aliyun.com \\ †chunlei-fan@aliyun.com \\ ${ }^{\ddagger}$ qunding@aliyun.com
}

Received December 26, 2017

\begin{abstract}
The chaotic system is widely used in chaotic cryptosystem and chaotic secure communication. In this paper, a universal method for designing the discrete chaotic system with any desired number of positive Lyapunov exponents is proposed to meet the needs of hyperchaotic systems in chaotic cryptosystem and chaotic secure communication, and three examples of eight-dimensional discrete system with chaotic attractors, eight-dimensional discrete system with fixed point attractors and eight-dimensional discrete system with periodic attractors are given to illustrate how the proposed methods control the Lyapunov exponents. Compared to the previous methods, the positive Lyapunov exponents are used to reconstruct a hyperchaotic system.
\end{abstract}

Keywords: Discrete chaotic system; Lyapunov exponents; Jacobi matrix; multistability.

\section{Introduction}

The Lyapunov exponent is a numerical characteristic that represents the average exponential divergence rate of adjacent trajectories in phase space, and it is one of the numerical characteristics used to determine chaotic behavior. The nonchaos fixed point attractors have negative Lyapunov exponents. The nonchaos periodic or limit cycle attractors have nonpositive Lyapunov exponents. The chaotic attractors have positive Lyapunov exponents, so the chaotic system will have at least a positive Lyapunov exponent.

With the rapid development of network technology, information security has attracted more and more attention. The chaotic cryptosystem and chaotic secure communication have been studied deeply Pan et al., 2012; Ding \& Wang, 2011;
Chen et al., 2004; Habutsu et al., 1991. The complex dynamic behavior of a chaotic system is an important guaranteed basis for information security in chaotic cryptosystem and chaotic secure communication. Therefore, the chaotic behavior of the chaotic system is of vital importance to determine the security of chaotic cryptosystem and chaotic secure communication. In recent years, the security defects of chaotic cryptosystem and chaotic secure communication with low-dimensional chaotic systems such as one-dimensional and two-dimensional are gradually emerging Solak, 2004; Zhu et al., 2011; Bhatnagar \& Wu, 2012; Xie et al., 2017]. Many high-dimensional chaotic systems have been proposed to solve these problems. However, chaotic cryptosystem and chaotic secure communication based on high-dimensional chaotic systems also

\footnotetext{
¥Author for correspondence

This is an Open Access article published by World Scientific Publishing Company. It is distributed under the terms of the Creative Commons Attribution 4.0 (CC-BY) License. Further distribution of this work is permitted, provided the original work is properly cited.
} 
cannot guarantee their safety. If low-dimensional chaotic systems and high-dimensional chaotic systems have the same number of positive Lyapunov exponents, the complexity of their chaotic behavior will also be quite similar.

The hyperchaotic system plays an important role in chaotic cryptosystem and chaotic secure communication because it has multiple positive Lyapunov exponents. In the $m$-dimensional continuous hyperchaotic system, the number of positive Lyapunov exponents is not more than $m-2$. In the $m$-dimensional discrete hyperchaotic system, the upper limit number of the positive Lyapunov exponents is $m$. Therefore, under the same dimensions, the nonlinear dynamic behavior of the discrete hyperchaotic system is more complex.

Although many design methods of high-dimensional hyperchaotic systems have been proposed, the results reflect that most of the hyperchaotic systems design methods are mainly aimed at threedimensional or four-dimensional hyperchaotic systems Rössler, 1979; Rech \& Albuquerque, 2009; Barboza, 2007]. The five-dimensional or six-dimensional hyperchaotic systems can be designed by state inverse control method $\mathrm{Hu}, 2009 ; \mathrm{Li}$ et al. 2005; Wang \& Wang, 2008; Chen et al., 2006]. Some of the hyperchaotic systems are designed by the method of parameter perturbation and coupling Kapitaniak et al., 1994; Cafagna \& Grassi, 2003; Li et al., 2010; Wang et al., 2008]. These methods can only work in certain dimensional chaotic systems, and cannot be used as general method for designing hyperchaotic systems. Although a designing method of high-dimensional chaotic system is proposed, it can only ensure that the designed system is a chaotic system rather than a hyperchaotic system [Kwok \& Tang, 2007].

In 1996, Chen proposed the chaos feedback control method to control the number of positive Lyapunov exponents in the discrete chaotic system Chen \& Lai, 1996. On this basis, Yu proposed a universal design of a discrete chaotic system with the most positive Lyapunov exponents Lin et al., 2015, and the design result of the eight-dimensional discrete chaotic system is given. Although the chaos feedback control method can well control the number of positive Lyapunov exponents, the selection of the feedback coefficient and the design of the poles are very complex. The value of the positive Lyapunov exponents grows very slowly with the increase of feedback control parameters. The large feedback coefficients are used to achieve a small increase in the positive Lyapunov exponents. For example, the three feedback coefficients are $\xi_{1}=$ $1.0 \times 10^{9}, \xi_{1}=1.2 \times 10^{9}, \xi_{1}=7.5 \times 10^{8}$ and the eight Lyapunov exponents are $L E_{1}=15.49, L E_{2}=$ $15.48, L E_{3}=14.43, L E_{4}=14.40, L E_{5}=14.18$, $L E_{6}=14.12, L E_{7}=7.23, L E_{8}=6.45$ Lin et al. , 2015]. In the ideal case, the value of $L E_{7}$ and $L E_{8}$ also should be close to 14 or 15 .

In the era of big data, the complex dynamic behavior of the chaotic system is no longer the only index for chaotic cryptosystem and chaotic secure communication. More and more attention has been paid to the computing complexity of the chaotic system and the efficiency of hardware implementation. Therefore, the large feedback coefficient limits the application of this kind of discrete chaotic system in the field of chaotic cryptosystem and chaotic secure communication.

Multistability exists in various nonlinear dynamic systems. Different initial values have different effects on the nonlinear dynamic system. It is very unsafe to use the chaotic system to realize chaotic cryptosystem and chaotic secure communication if a discrete chaotic system shows a nonchaotic behavior under some initial values. Therefore, chaotic systems in chaotic cryptosystem and chaotic secure communication need to avoid the nonchaotic state.

In this paper, a design method of discrete chaotic systems with any desired number of positive Lyapunov exponents is proposed. The design process of the proposed scheme is completely opposite to that of other existing methods. The given positive Lyapunov exponents are used to reconstruct the chaos system by the proposed scheme. In Sec. 2, a high-dimensional discrete chaotic design method with any desired number of positive Lyapunov exponents is introduced. Section 3 gives three concrete examples including eight-dimensional discrete system with chaotic attractors, eight-dimensional discrete system with fixed point attractors and eight-dimensional discrete system with periodic attractors. The conclusion is given in the last section.

\section{A Discrete Chaotic Design Method for any Desired Number of Positive Lyapunov Exponents}

\subsection{Calculating the Lyapunov exponents via Jacobi method}

The Jacobi method is a common way to calculate Lyapunov exponents. Suppose the $m$-dimensional 
discrete chaotic map is

$$
\left\{\begin{aligned}
x_{1}(n+1) & =f_{1}\left(x_{1}, x_{2}, \ldots, x_{m}\right) \\
x_{2}(n+1) & =f_{2}\left(x_{1}, x_{2}, \ldots, x_{m}\right) \\
& \vdots \\
x_{m}(n+1) & =f_{m}\left(x_{1}, x_{2}, \ldots, x_{m}\right) .
\end{aligned}\right.
$$

The Jacobi matrix of (1) is

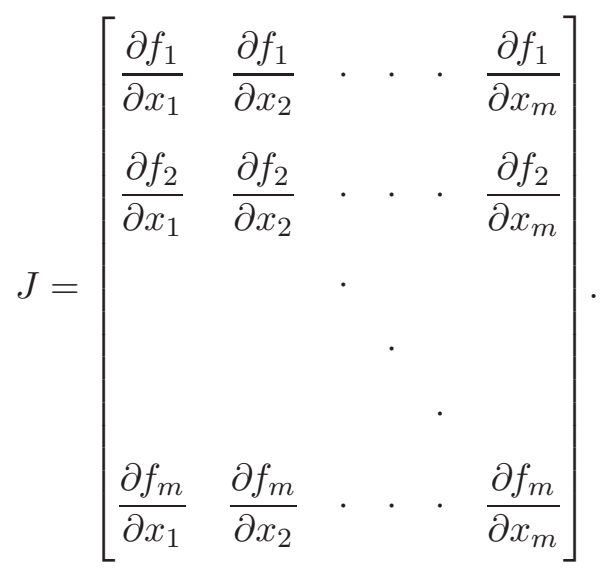

The $i$ th iteration Jacobi matrix is $J_{i}=J\left(x_{1}(i)\right.$, $\left.x_{2}(i), \ldots, x_{m}(i)\right), i=0,1,2, \ldots$ The matrix $P$ is

$$
A=\left[\begin{array}{cccccccc}
a_{1,1} & a_{1,2} & \cdot & \cdot & \cdot & a_{1, m-2} & a_{1, m-1} & a_{1, m} \\
a_{2,1} & a_{2,2} & \cdot & \cdot & \cdot & a_{2, m-2} & a_{2, m-1} & a_{2, m} \\
\cdot & \cdot & \cdot & \cdot & \cdot & \cdot & \cdot & \cdot \\
\cdot & \cdot & & \cdot & \cdot & \cdot & \cdot \\
\cdot & \cdot & & & \cdot & \cdot & \cdot & \cdot \\
a_{m, 1} & a_{m, 2} & \cdot & \cdot & \cdot & a_{m, m-2} & a_{m, m-1} & a_{m, m}
\end{array}\right] .
$$

For no nonlinear term in (5), the Jacobi matrix is $A$. Therefore, $P=A^{n}$. Suppose the $m$ eigenvalues of the matrix $A$ are $\lambda_{0}, \lambda_{1}, \ldots, \lambda_{m}$. The $m$ Lyapunov exponents are

$$
\left\{\begin{array}{c}
L E_{1}=\frac{1}{n} \ln \left|\lambda_{1}\right|^{n}=\ln \left|\lambda_{1}\right| \\
L E_{2}=\frac{1}{n} \ln \left|\lambda_{2}\right|^{n}=\ln \left|\lambda_{2}\right| \\
\vdots \\
L E_{m}=\frac{1}{n} \ln \left|\lambda_{m}\right|^{n}=\ln \left|\lambda_{m}\right| .
\end{array}\right.
$$

Therefore, the eigenvalues of the matrix $A$ determine the Lyapunov exponents in (5) completely. However, the eigenvalues of the parameter matrix $A$ defined as

$$
P=J_{0} J_{1} \cdots J_{n}
$$

Suppose the $m$ eigenvalues of the matrix $P$ are $\lambda_{0}$, $\lambda_{1}, \ldots, \lambda_{m}$. Then, the Lyapunov exponent of (1) is $L E_{1}=\frac{1}{n} \ln \left|\lambda_{1}\right|, L E_{2}=\frac{1}{n} \ln \left|\lambda_{2}\right|, \ldots, L E_{m}=\frac{1}{n} \ln \left|\lambda_{m}\right|$.

From the Jacobi method, the Lyapunov exponents are determined completely by the eigenvalues of Jacobi matrix. So, is it possible to restructure (3) from (4), and restructure (3) from (2), and ultimately restructure the chaotic system (11).

\subsection{Discrete chaotic systems modeling}

With converse thinking, a discrete chaotic system model is studied in detail, and it is defined as

$$
S_{n+1}=A S_{n},
$$

where $S_{n}$ is the vector state $\left(x_{1}(n), x_{2}(n), x_{3}(n), \ldots\right.$, $\left.x_{m}(n)\right)^{T}$, and parameter matrix $A$ is a constant matrix.

cannot guarantee that (5) is a chaotic system, because the parameter matrix $A$ cannot guarantee that (5) is bounded. The boundedness of (5) is guaranteed by congruent function in this paper. Therefore, (5) can be transformed into (86):

$$
S_{n+1}=A S_{n} \bmod c
$$

where the elements $x_{i}(n), i=1,2,3, \ldots, m$ are in the interval $[0, c]$.

\subsection{Route for reconstructing discrete chaotic system via positive Lyapunov exponents}

Some properties of the matrix can be used to reconstruct the discrete chaotic system Roger \& Charles, 1985.]. 
Definition 2.1. For $m \times m$ matrix $A$ and $B$, the matrix $B$ is said to be similar to the matrix $A$ if there exists a full rank matrix such that $B=q^{-1} A q$.

Proposition 1. Similar matrix $A$ and $B$ must have the same eigenvalues.

Proof. $\operatorname{det}(A-\lambda I)=\operatorname{det}\left(q B q^{-1}-\lambda q I q^{-1}\right)=$ $\operatorname{det}(q) \cdot \operatorname{det}(B-\lambda I) \cdot \operatorname{det}\left(q^{-1}\right)=\operatorname{det}(B-\lambda I)$.

Proposition 2. $\lambda_{1}, \lambda_{2}, \ldots, \lambda_{m}$ are the $m$ eigenvalues of the matrix $A$ if the matrix $\Lambda$ is similar to the matrix A, where

$$
\Lambda=\left[\begin{array}{llllll}
\lambda_{1} & & & & & \\
& \lambda_{2} & & & \\
& & \cdot & & \\
& & & \cdot & & \\
& & & \cdot & \\
& & & & \lambda_{m}
\end{array}\right] .
$$

Proof. For $\operatorname{det}(A-\lambda \Lambda)=0, \lambda_{1}, \lambda_{2}, \ldots, \lambda_{m}$ are the $m$ exponents of the matrix $\Lambda$. From Proposition $11 \lambda_{1}, \ldots, \lambda_{m}$ are also the $m$ exponents of the matrix $A$.

Theorem 1. The necessary and sufficient condition for diagonalization of $m$ order matrix $A$ is that $A$ has $m$ linear independent eigenvectors.

Proof. Suppose there exists a full rank $m \times m$ matrix $q$ such that $q^{-1} A q=\Lambda$, where matrix $\Lambda$ is a diagonal matrix. The matrix $q$ can be expanded to be a column vector $q=\left(q_{1}, q_{2}, \ldots, q_{n}\right)$. For $q^{-1} A q=\Lambda, A q=q \Lambda$, and it also can be represented by column vectors:

$$
\begin{aligned}
& A\left(q_{1}, q_{2}, \ldots, q_{m}\right) \\
& \quad=\left(q_{1}, q_{2}, \ldots, q_{m}\right)\left[\begin{array}{lllll}
\lambda_{1} & & & & \\
& \lambda_{2} & & & \\
& & \cdot & & \\
& & \cdot & \\
& & & & \\
& & & \lambda_{m}
\end{array}\right] \\
& =\left(\lambda_{1} q_{1}, \lambda_{2} q_{2}, \ldots, \lambda_{m} q_{m}\right) .
\end{aligned}
$$

Therefore, $A q=\lambda_{i} p_{i}(i=1,2, \ldots, m) . \lambda_{i}$ is the eigenvalue of matrix $A$. The column vectors in matrix is the eigenvectors corresponding to the eigenvalue $\lambda_{i}$. For matrix $q$ is a full rank matrix, the column vectors $q_{1}, q_{2}, \ldots, q_{n}$ are linearly independent.

Instead, the matrix $A$ has $m$ eigenvalues, so the corresponding $m$ linear independent eigenvectors can be calculated. These $m$ linear independent eigenvectors can be constructed as full rank matrix such that $A q=q \Lambda$. The matrix $q$ is invertible, because column vectors $q_{1}, q_{2}, \ldots, q_{n}$ are linearly independent. Multiplying both sides by $q^{-1}$ in equation $A q=q \Lambda$ yields $q^{-1} A q=\Lambda$.

In summary, a method for constructing a discrete chaotic system with any desired number of Lyapunov exponents is shown as follows:

(i) Given the positive Lyapunov exponents $L E_{1}$, $L E_{2}, \ldots, L E_{m}$, then calculate the eigenvalues $\lambda_{1}=e^{L E_{1}}, \lambda_{2}=e^{L E_{2}}, \ldots, \lambda_{m}=e^{L E_{m}}$. The diagonal matrix $\Lambda$ based on eigenvalues is constructed.

$$
\Lambda=\left[\begin{array}{ccccc}
L E_{1} & & & & \\
& L E_{2} & & & \\
& & \cdot & & \\
& & \cdot & & \\
& & & \cdot & \\
& & & & L E_{m}
\end{array}\right] .
$$

(ii) Design a $m \times m$ nonsingular matrix $q$.

(iii) Calculate parameter matrix $A=q \Lambda q^{-1}$, and reconstruct the discrete chaotic system via (8).

\section{Examples}

\subsection{The eight-dimensional discrete system with chaotic attractors}

The design of the eight-dimensional discrete chaotic system is as follows:

(i) Given eight eigenvalues that are greater than 1 , such as $53,32,78,45,56,23,39,89$, and $c$ is defined as 1 . The corresponding rounded Lyapunov exponents are $3.97,3.47,4.36,3.81,4.03,3.14$, $3.66,4.49$. 
(ii) A simple nonsingular matrix $q$ is defined as

$$
q=\left[\begin{array}{cccccc}
2 & 1 & \cdot & \cdot & \cdot & 1 \\
1 & 2 & \cdot & \cdot & \cdot & 1 \\
\cdot & \cdot & \cdot & & & \cdot \\
\cdot & \cdot & & \cdot & & \cdot \\
\cdot & \cdot & & & \cdot & \cdot \\
1 & 1 & \cdot & \cdot & \cdot & 2
\end{array}\right],
$$

where elements $q(i, i)=2, i=1,2,3, \ldots, m$, the other elements are all 1 . It is easy to prove that matrix $q$ is a nonsingular matrix. When $m=8$, $q$ can be defined as

$$
q=\left[\begin{array}{llllllll}
2 & 1 & 1 & 1 & 1 & 1 & 1 & 1 \\
1 & 2 & 1 & 1 & 1 & 1 & 1 & 1 \\
1 & 1 & 2 & 1 & 1 & 1 & 1 & 1 \\
1 & 1 & 1 & 2 & 1 & 1 & 1 & 1 \\
1 & 1 & 1 & 1 & 2 & 1 & 1 & 1 \\
1 & 1 & 1 & 1 & 1 & 2 & 1 & 1 \\
1 & 1 & 1 & 1 & 1 & 1 & 2 & 1 \\
1 & 1 & 1 & 1 & 1 & 1 & 1 & 2
\end{array}\right]
$$

and the rounded inverse matrix $q^{-1}$ is

$$
q^{-1}=\left[\begin{array}{rrrrrrrr}
0.89 & -0.11 & -0.11 & -0.11 & -0.11 & -0.11 & -0.11 & -0.11 \\
-0.11 & 0.89 & -0.11 & -0.11 & -0.11 & -0.11 & -0.11 & -0.11 \\
-0.11 & -0.11 & 0.89 & -0.11 & -0.11 & -0.11 & -0.11 & -0.11 \\
-0.11 & -0.11 & -0.11 & 0.89 & -0.11 & -0.11 & -0.11 & -0.11 \\
-0.11 & -0.11 & -0.11 & -0.11 & 0.89 & -0.1111 & -0.11 & -0.11 \\
-0.11 & -0.11 & -0.11 & -0.11 & -0.11 & 0.89 & -0.11 & -0.11 \\
-0.11 & -0.11 & -0.11 & -0.11 & -0.11 & -0.11 & 0.89 & -0.11 \\
-0.11 & -0.11 & -0.11 & -0.11 & -0.11 & -0.11 & -0.11 & 0.89
\end{array}\right] .
$$

(iii) The parameter matrix is

$$
A=q \Lambda q^{-1}=\left[\begin{array}{rrrrrrrr}
54.52 & -19.48 & 26.52 & -6.48 & 4.52 & -28.48 & -12.48 & 37.52 \\
3.83 & 14.83 & 28.83 & -4.17 & 6.83 & -26.17 & -10.17 & 39.83 \\
-1.23 & -22.23 & 101.77 & -9.23 & 1.77 & -31.23 & -15.23 & 34.77 \\
2.40 & -18.60 & 27.40 & 39.40 & 5.40 & -27.60 & -11.60 & 38.40 \\
1.19 & -19.81 & 26.19 & -6.81 & 60.19 & -28.81 & -12.81 & 37.19 \\
4.82 & -16.18 & 29.82 & -3.18 & 7.82 & -2.18 & -9.18 & 40.82 \\
3.06 & -17.94 & 28.06 & -4.94 & 6.06 & -26.94 & 28.06 & 39.06 \\
-2.44 & -23.44 & 22.56 & -10.44 & 0.56 & -32.44 & -16.44 & 122.56
\end{array}\right] .
$$

The matrix $A$ is a rounded parameter matrix, because matrix $q^{-1}$ is a rounded inverse matrix. Therefore, the eigenvalues are calculated from the matrix $A$ again. The rounded eight eigenvalues of matrix $A$ are $90.01,78.77,23.20,32.28,39.34,45.42$, $53.45,56.67$, and they are very close to the prearranged values in Step (i). The rounded eight Lyapunov exponents are calculated as 4.5, 4.37, 3.14, $3.47,3.67,3.82,3.98,4.04$. The phase diagram of the chaotic attractors is shown in Fig. 1.
The features of the chaos can be expressed by the track of the chaos attractor in phase space. In Fig. 1, the whole phase space is fully filled with chaotic trajectories. The output time series is shown in Fig. 2 from which, the eightdimensional hyperchaotic system consisting of the parameter matrix (12) can output eight different pseudo-random sequences simultaneously. The eight-dimensional discrete system is in the chaotic 


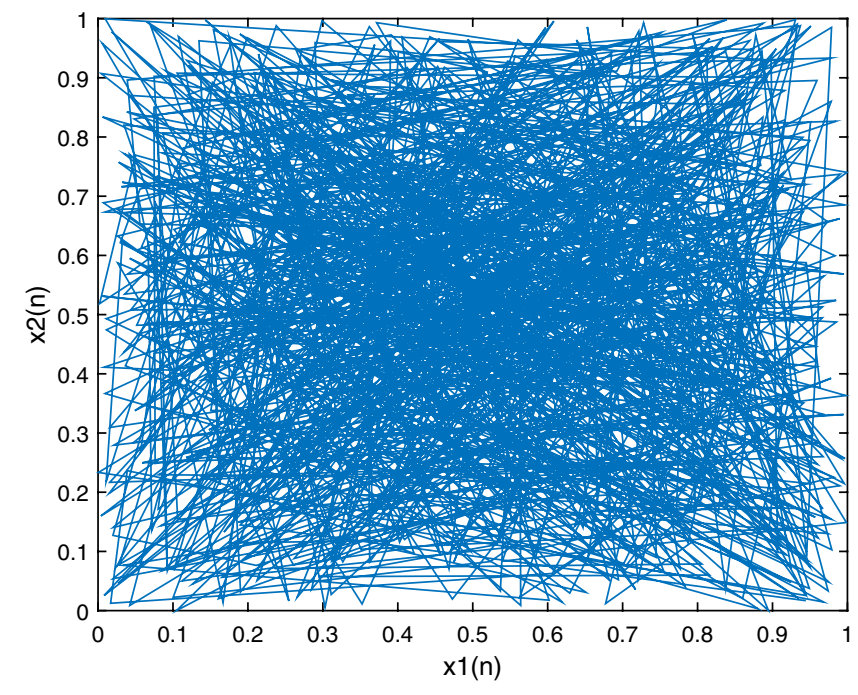

(a)

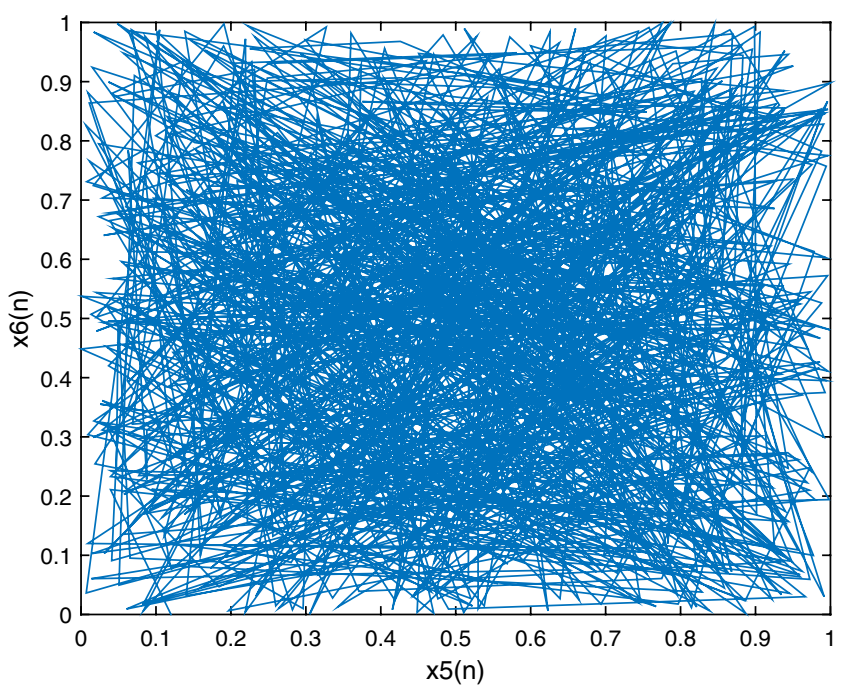

(c)

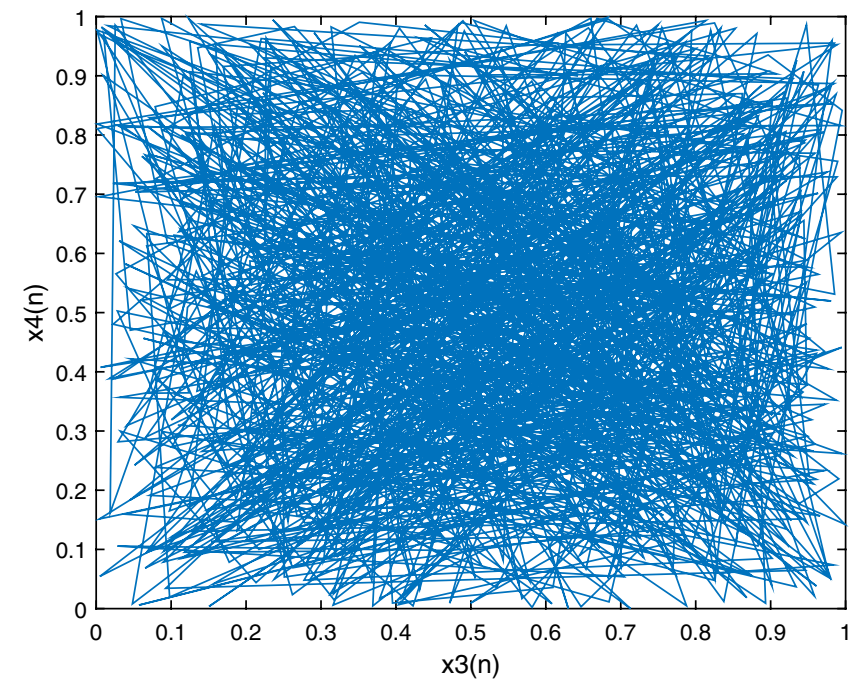

(b)

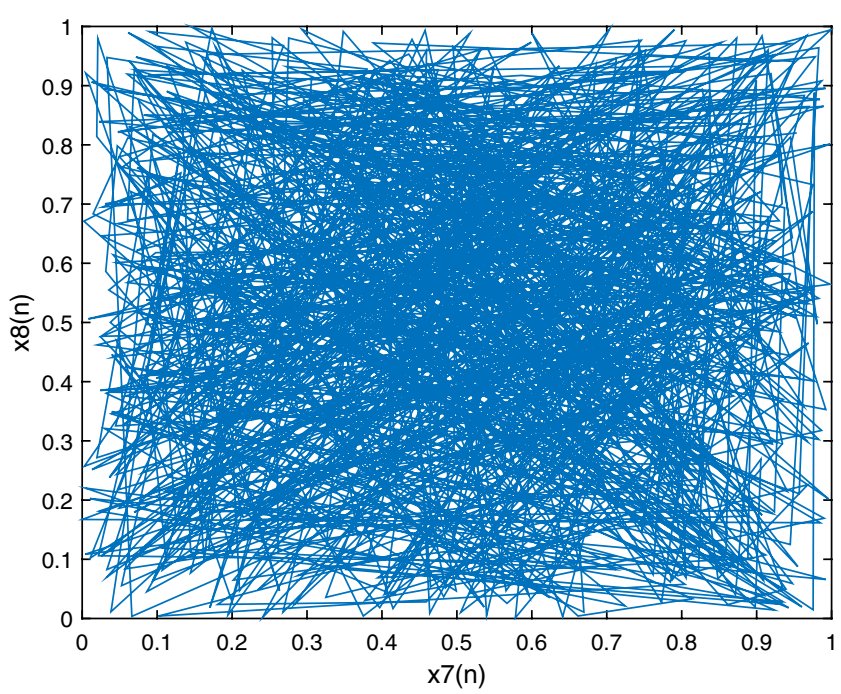

(d)

Fig. 1. The phase diagram of chaotic attractors, (a) $x_{1}(n)-x_{2}(n)$, (b) $x_{3}(n)-x_{4}(n)$, (c) $x_{5}(n)-x_{6}(n)$, (d) $x_{7}(n)-x_{8}(n)$, (e) $x_{1}(n)-x_{2}(n)-x_{3}(n)$, (f) $x_{4}(n)-x_{5}(n)-x_{6}(n)$ and $(\mathrm{g}) x_{6}(n)-x_{7}(n)-x_{8}(n)$. 


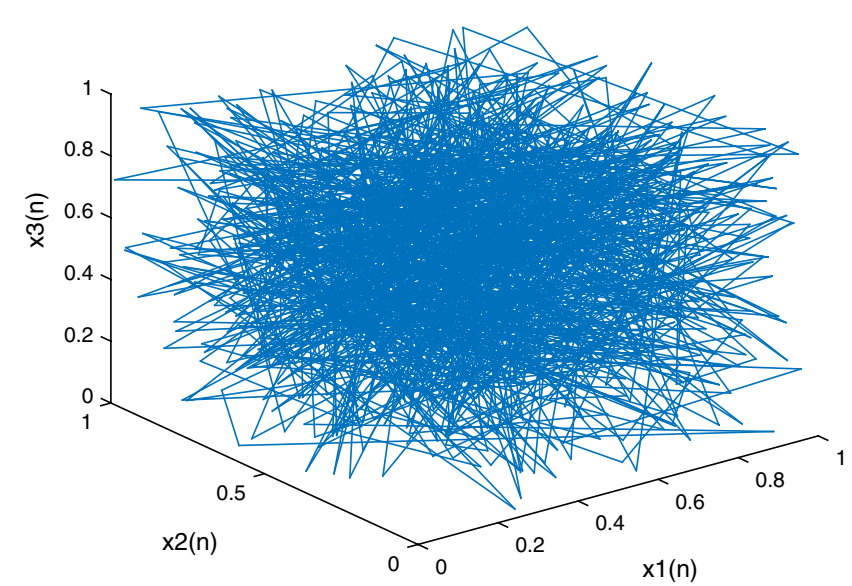

(e)

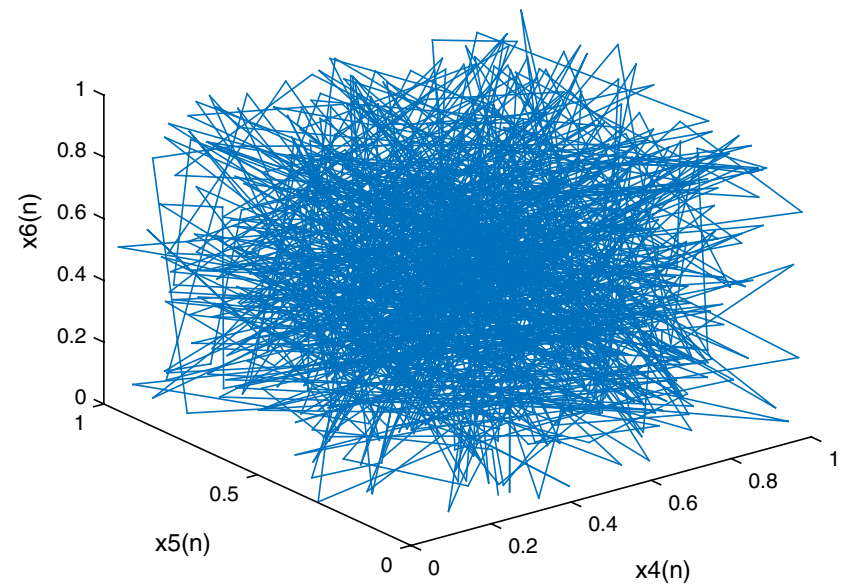

(f)

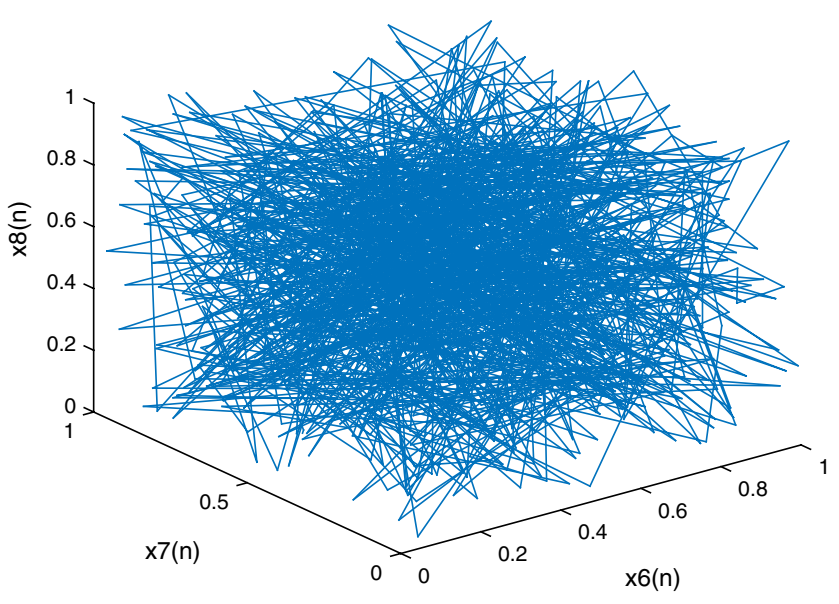

(g)

Fig. 1. (Continued) 


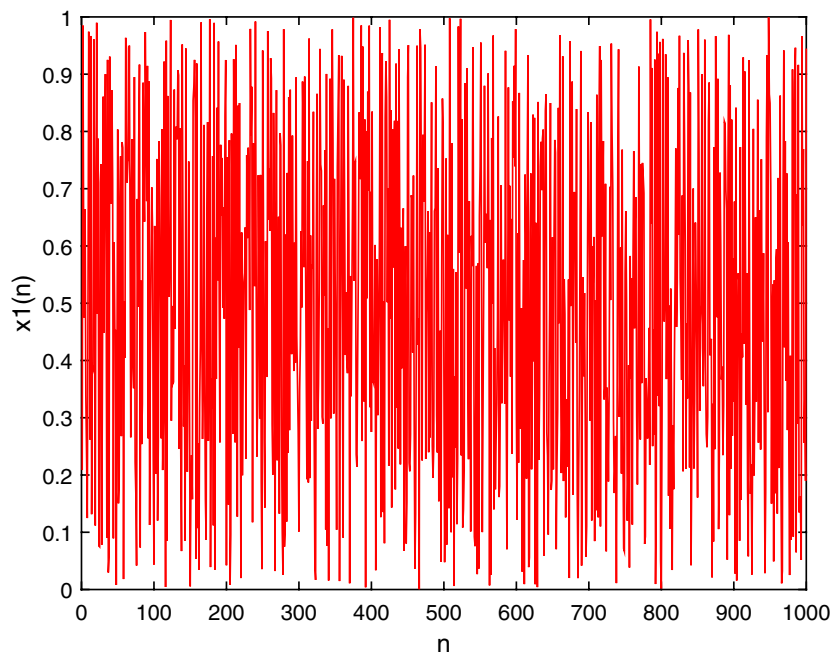

(a)

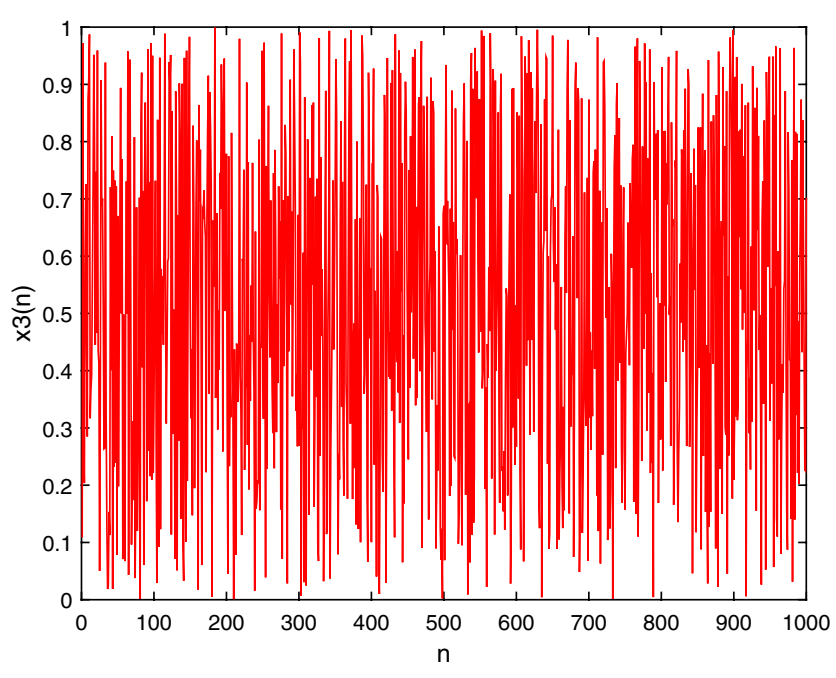

(c)

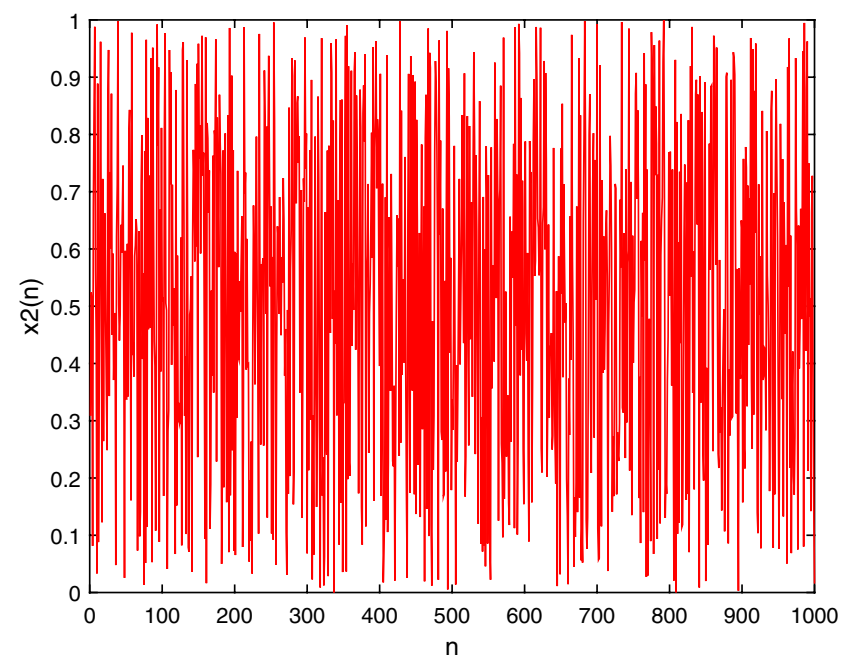

(b)

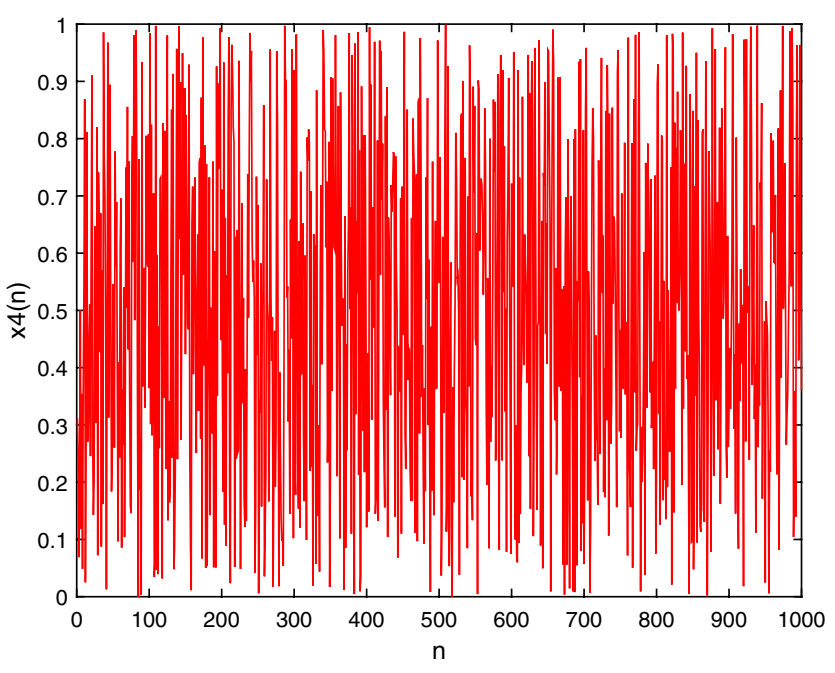

(d)

Fig. 2. The plots of output time series, (a) $x_{1}(n),(\mathrm{b}) x_{2}(n),(\mathrm{c}) x_{3}(n),(\mathrm{d}) x_{4}(n),(\mathrm{e}) x_{5}(n),(\mathrm{f}) x_{6}(n),(\mathrm{g}) x_{7}(n)$ and $(\mathrm{h}) x_{8}(n)$. 


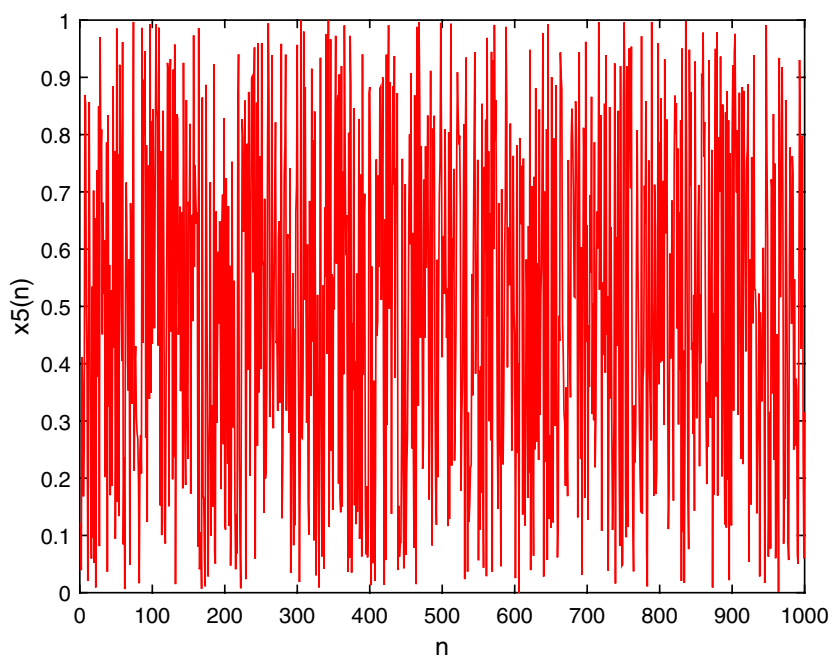

(e)

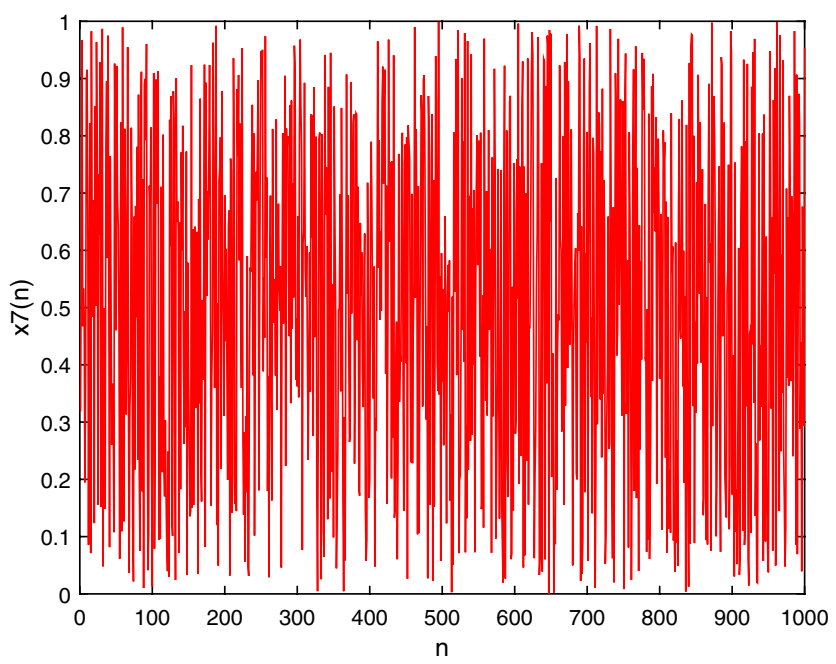

(g)

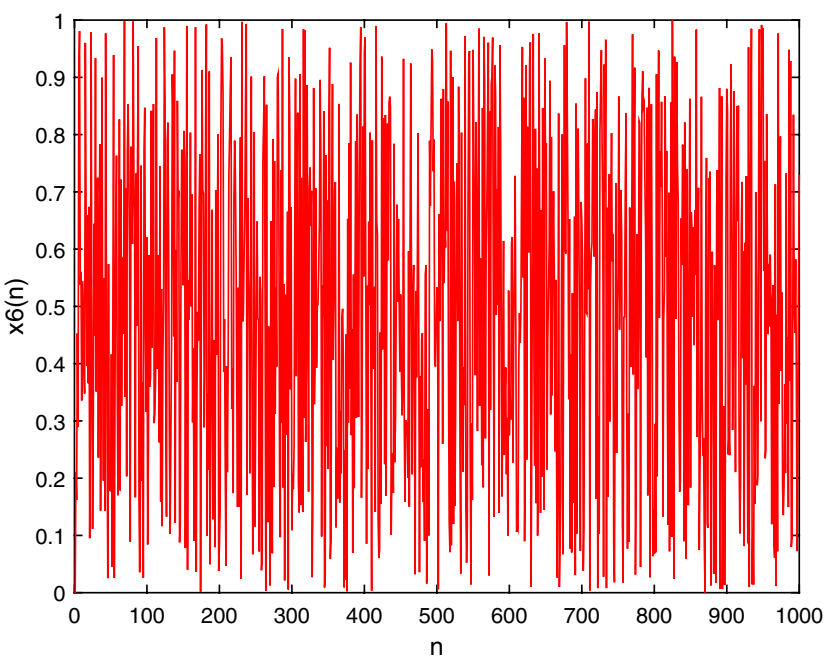

(f)

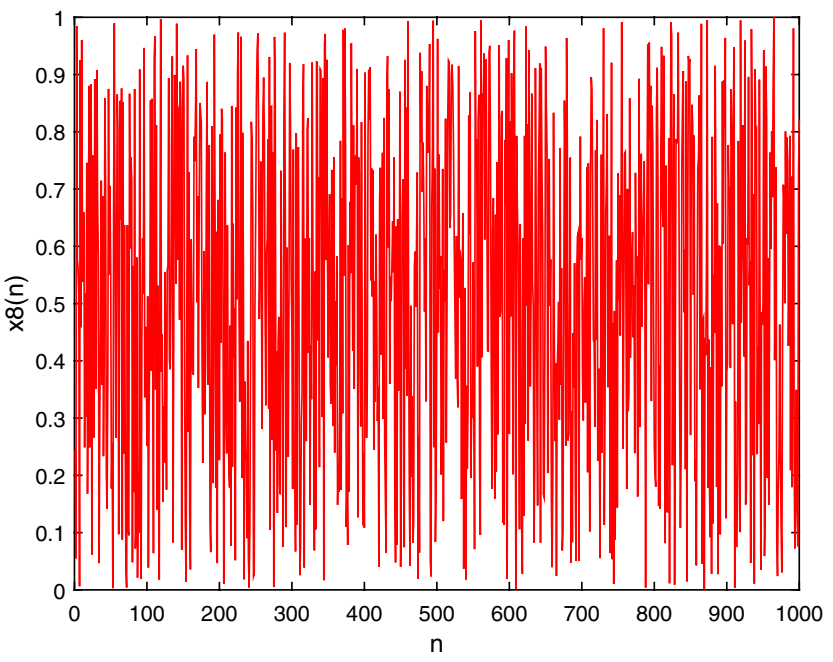

(h)

Fig. 2. (Continued) 
state for all initial values since the Jacobi matrix is independent of the output time series. The $m$ dimensional chaotic system (8) also has the same properties. In chaotic cryptosystem and chaotic secure communication based on this chaotic system, there will be no hidden danger of nonchaotic state.

\subsection{The eight-dimensional discrete system with fixed point attractors}

The design of the eight-dimensional discrete system with fixed point attractors is as follows:

(i) Given eight eigenvalues that are less than 1 , such as $0.53,0.32,0.78,0.45,0.56,0.23,0.39,0.89$, and $c$ is defined as 1 , the corresponding rounded Lyapunov exponents are $-0.63,-1.14,-0.25,-0.80$, $-0.58,-1.47,-0.94,-0.12$.

(ii) A simple nonsingular matrix $q$ is defined as (9).

(iii) The parameter matrix is

$$
A=q \Lambda q^{-1}=\left[\begin{array}{rrrrrrrl}
0.55 & -0.19 & 0.27 & -0.06 & 0.05 & -0.28 & -0.12 & 0.38 \\
0.04 & 0.15 & 0.29 & -0.04 & 0.07 & -0.26 & -0.1 & 0.4 \\
-0.01 & -0.22 & 1.02 & -0.09 & 0.02 & -0.31 & -0.15 & 0.35 \\
0.02 & -0.19 & 0.27 & 0.39 & 0.05 & -0.28 & -0.12 & 0.38 \\
0.01 & -0.20 & 0.26 & -0.07 & 0.60 & -0.29 & -0.13 & 0.37 \\
0.05 & -0.16 & 0.30 & -0.03 & 0.08 & -0.02 & -0.09 & 0.41 \\
0.03 & -0.18 & 0.28 & -0.05 & 0.06 & -0.27 & 0.28 & 0.39 \\
-0.02 & -0.23 & 0.23 & -0.10 & 0.01 & -0.32 & -0.16 & 1.23
\end{array}\right] .
$$

The rounded eight eigenvalues are $0.93,0.79$, $0.55+0.02 \mathrm{i}, 0.55-0.02 \mathrm{i}, 0.24,0.42,0.34,0.37$ and they are also very close to the prearranged values in Step (i). The rounded eight Lyapunov exponents are calculated as $-0.07,-0.24,-0.60,-0.60,-1.43$, $-0.87,-1.08,-0.99$. The phase diagram of the chaotic attractors is shown in Fig. 3. The phase diagram of the attractors finally approaches the fixed point, which can also be seen from the output time series. Although each output time series are different, they will eventually get close to a stable point. The output time series are shown in Fig. 4.

$$
A=q \Lambda q^{-1}=\left[\begin{array}{rrrrrrrr}
0.47 & -0.27 & 0.19 & 0.41 & -0.03 & -0.36 & -0.2 & 0.41 \\
-0.03 & 0.08 & 0.22 & 0.44 & 0 & -0.33 & -0.17 & 0.44 \\
-0.08 & -0.29 & 0.95 & 0.39 & -0.05 & -0.38 & -0.22 & 0.39 \\
-0.11 & -0.32 & 0.14 & 1.36 & -0.08 & -0.41 & -0.25 & 0.36 \\
-0.06 & -0.27 & 0.19 & 0.41 & 0.53 & -0.36 & -0.2 & 0.41 \\
-0.02 & -0.23 & 0.23 & 0.45 & 0.01 & -0.09 & -0.16 & 0.45 \\
-0.04 & -0.25 & 0.21 & 0.43 & -0.01 & -0.34 & 0.21 & 0.43 \\
-0.11 & -0.32 & 0.14 & 0.36 & -0.08 & -0.41 & -0.25 & 1.36
\end{array}\right]
$$




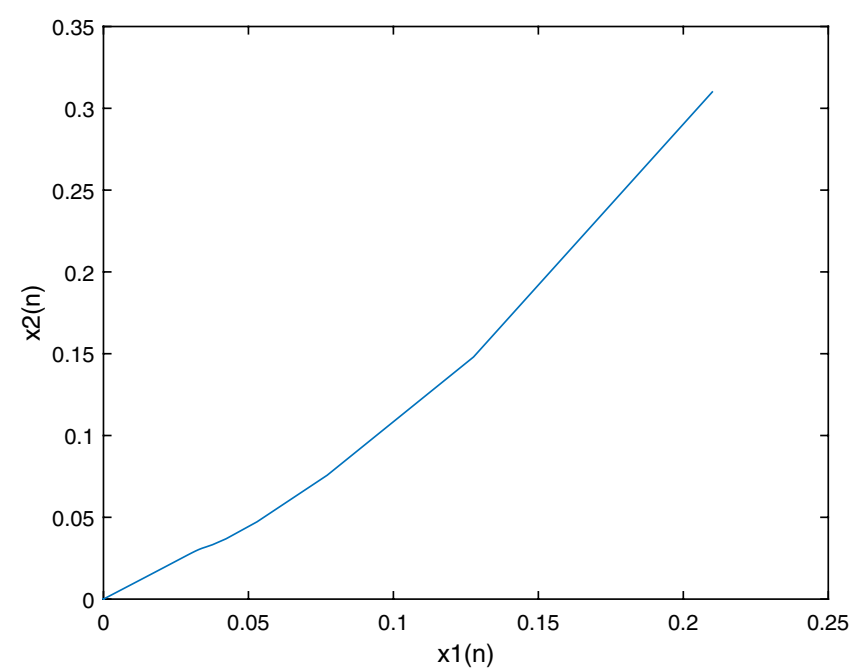

(a)

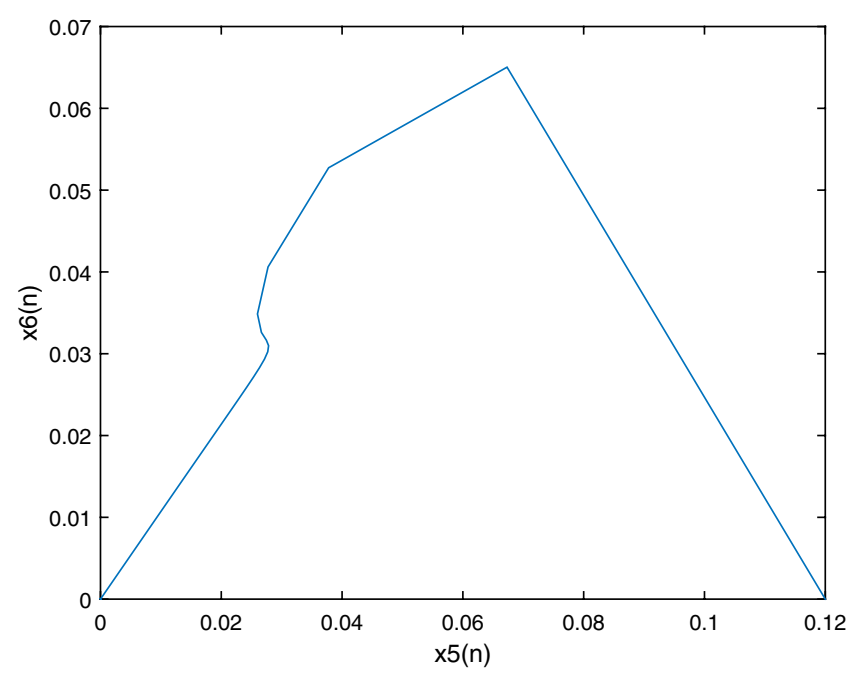

(c)

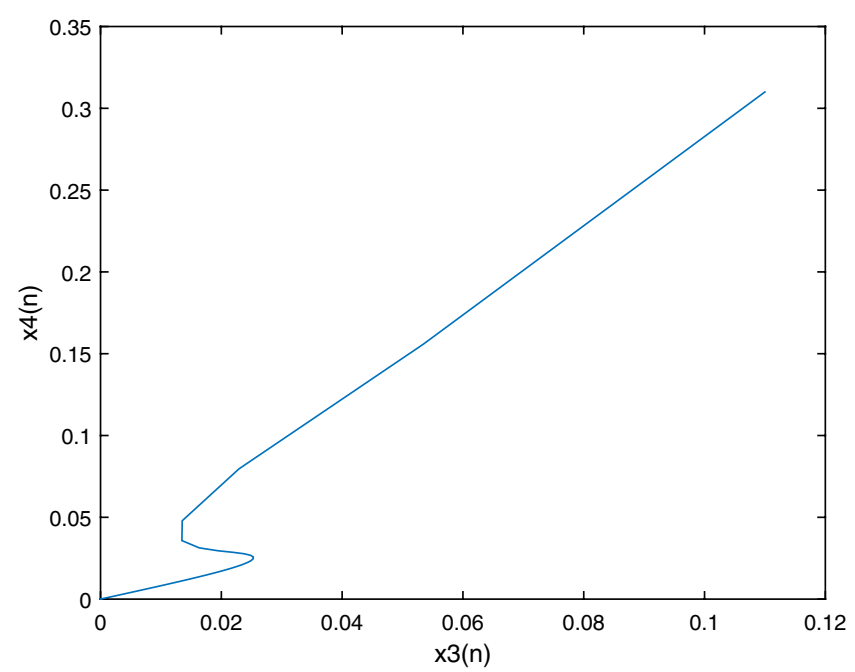

(b)

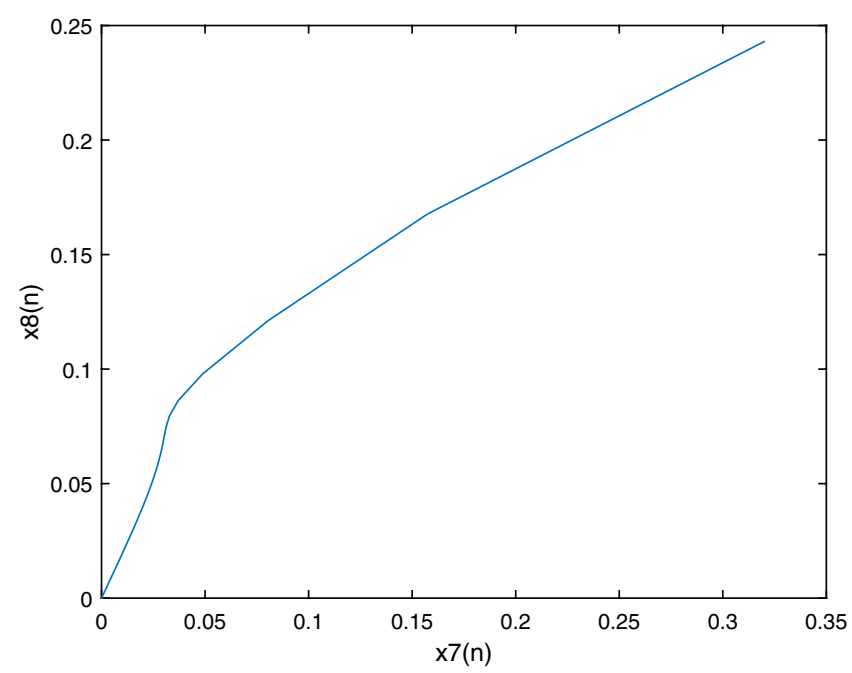

(d)

Fig. 3. The phase diagram of fixed point attractors, (a) $x_{1}(n)-x_{2}(n)$, (b) $x_{3}(n)-x_{4}(n),(\mathrm{c}) x_{5}(n)-x_{6}(n),(\mathrm{d}) x_{7}(n)-x_{8}(n)$, (e) $x_{1}(n)-x_{2}(n)-x_{3}(n)$, (f) $x_{4}(n)-x_{5}(n)-x_{6}(n)$ and (g) $x_{6}(n)-x_{7}(n)-x_{8}(n)$. 
C. Wang et al.

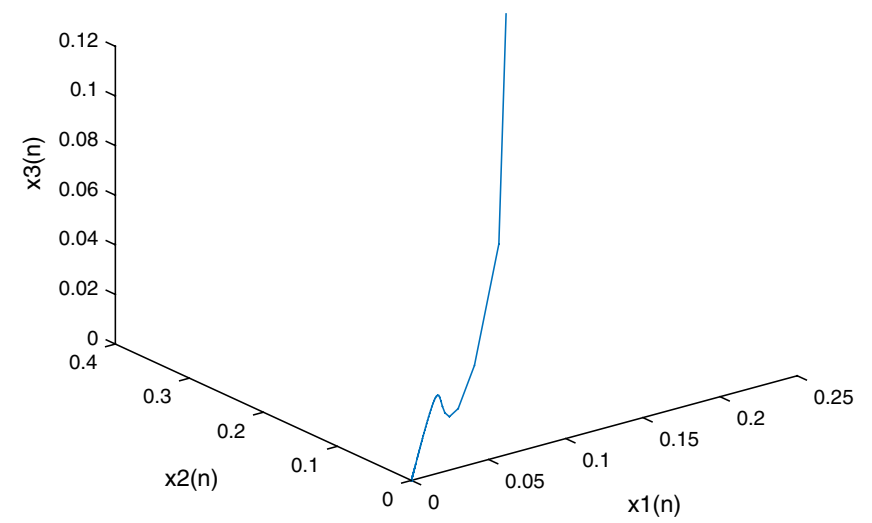

(e)

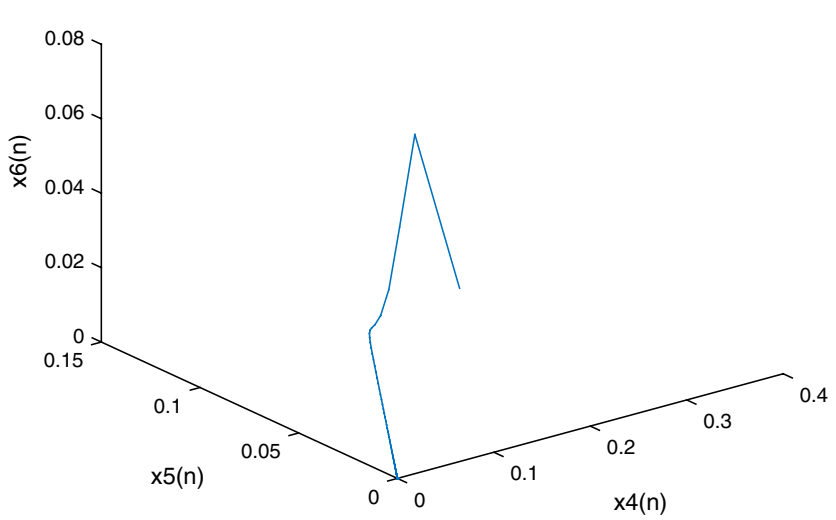

(f)

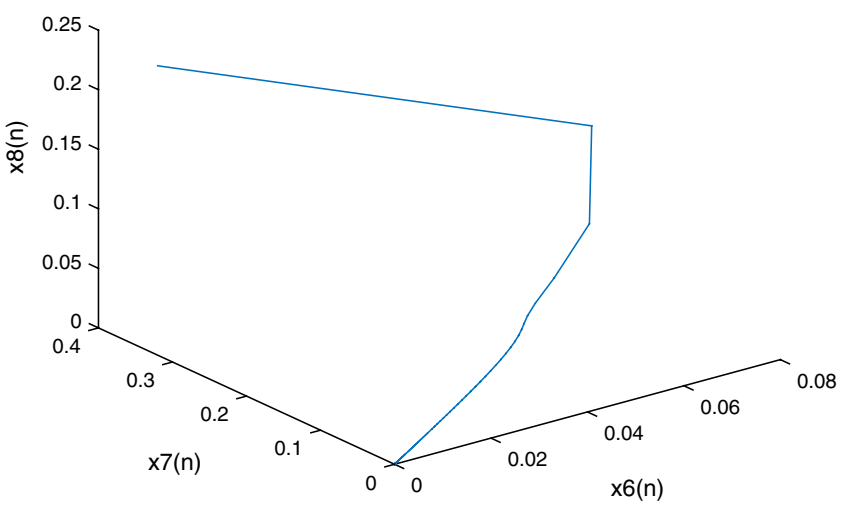

(g)

Fig. 3. (Continued)

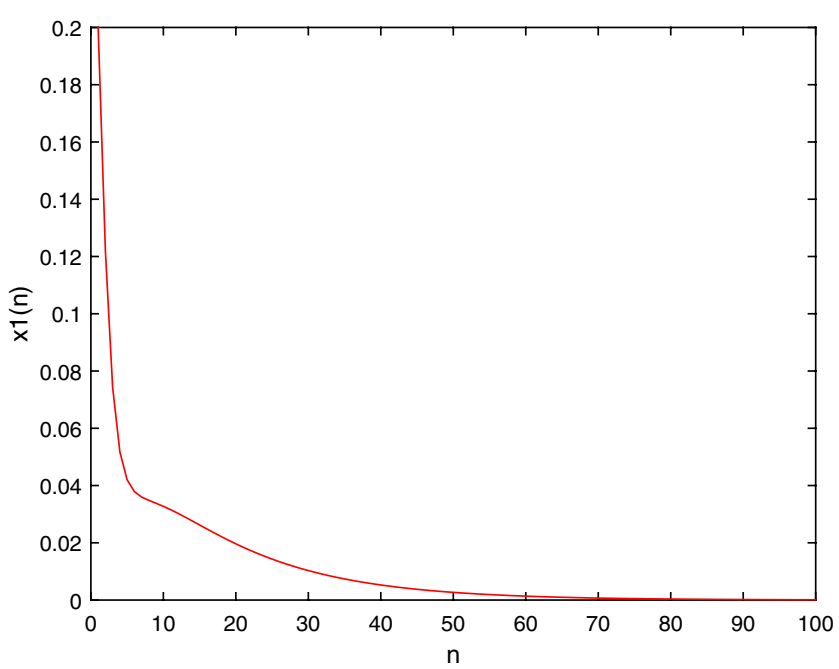

(a)

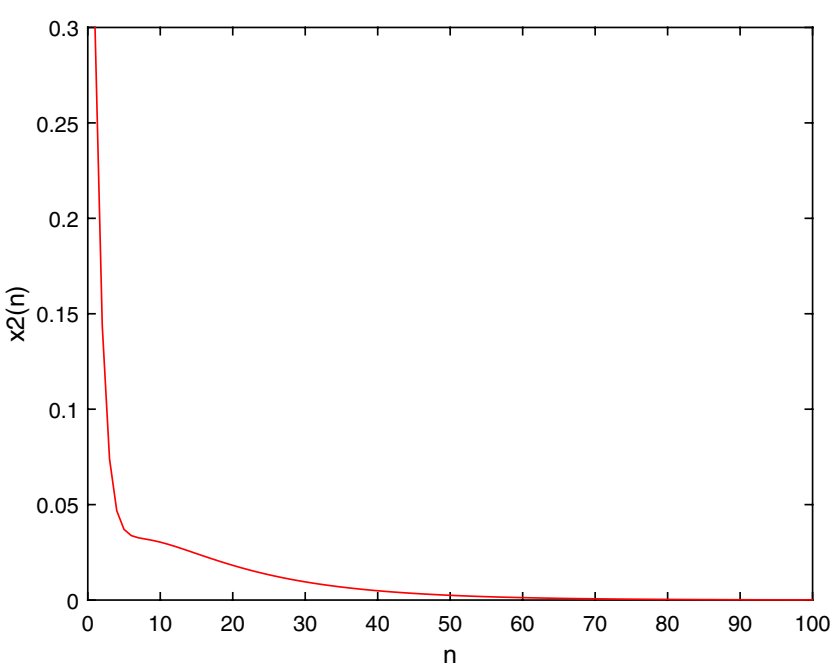

(b)

Fig. 4. The plots of output time series, (a) $x_{1}(n)$, (b) $x_{2}(n)$, (c) $x_{3}(n)$, (d) $x_{4}(n)$, (e) $x_{5}(n),(\mathrm{f}) x_{6}(n),(\mathrm{g}) x_{7}(n)$ and (h) $x_{8}(n)$. 
Constructing Discrete Chaotic Systems with Positive Lyapunov Exponents

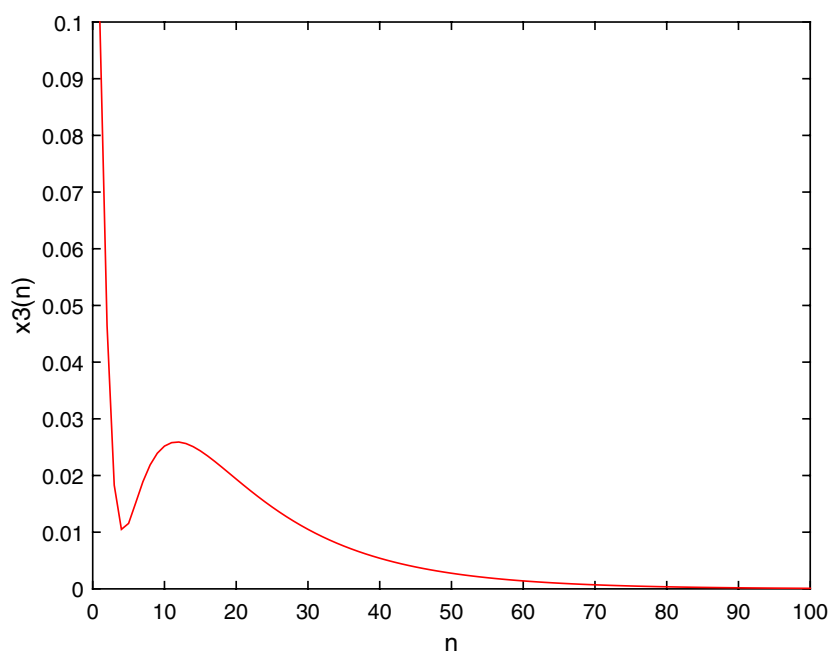

(c)

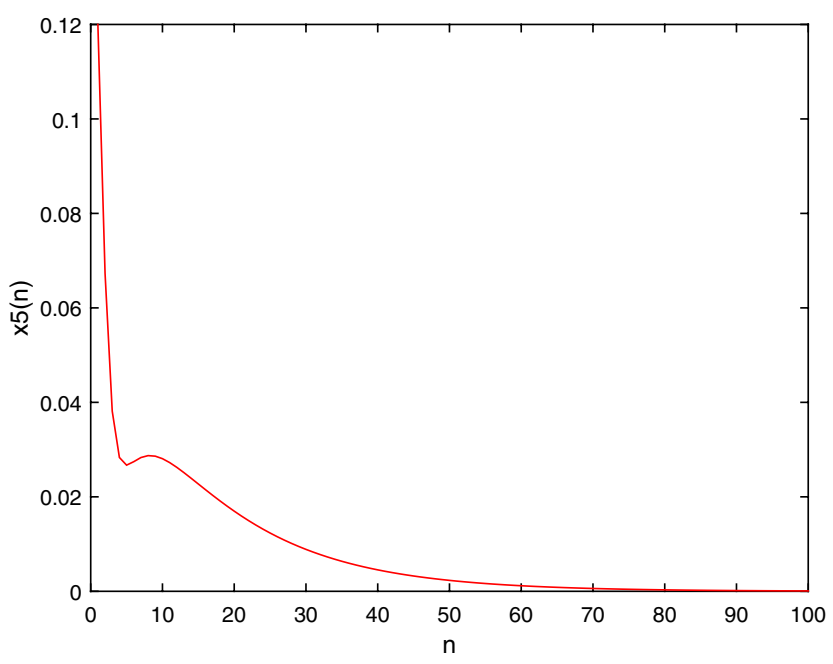

(e)

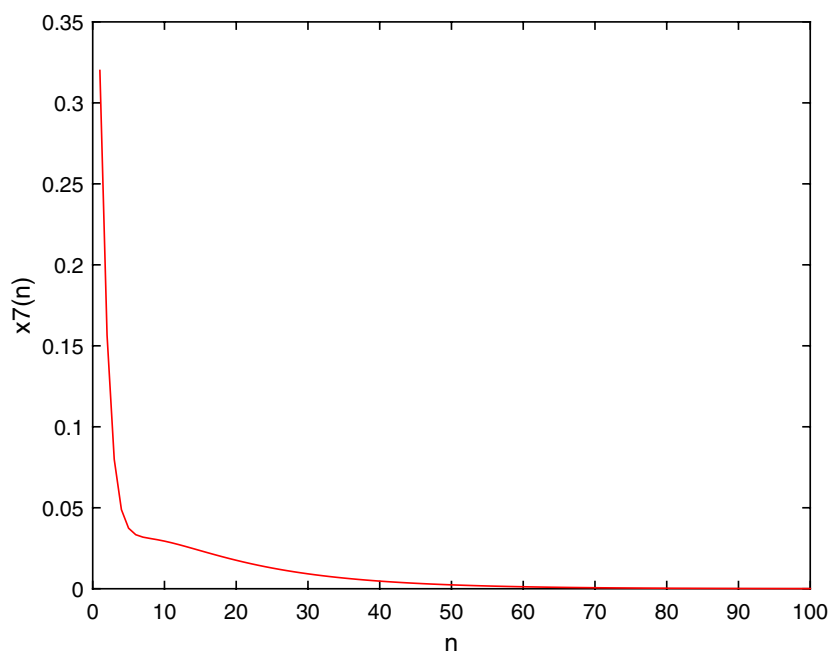

(g)

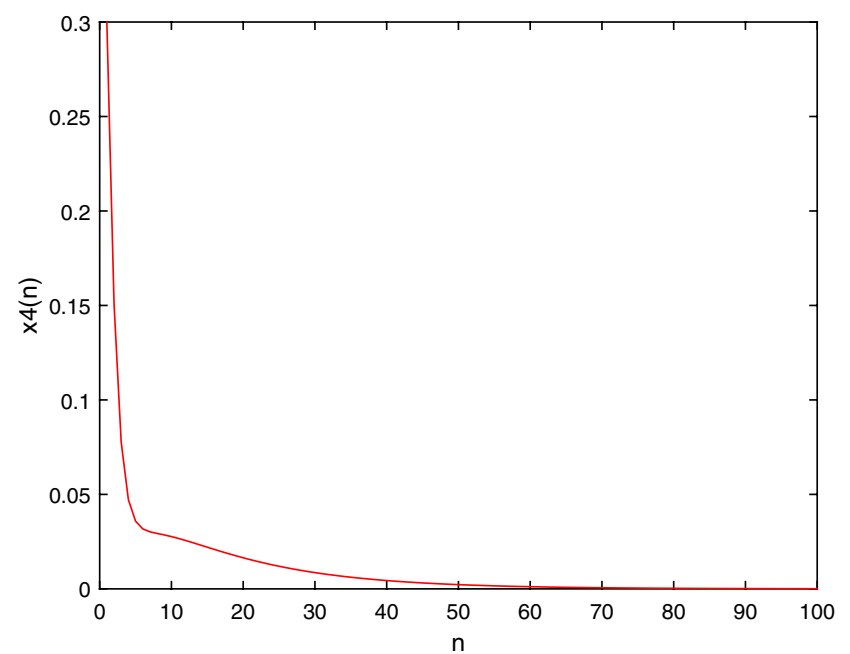

(d)

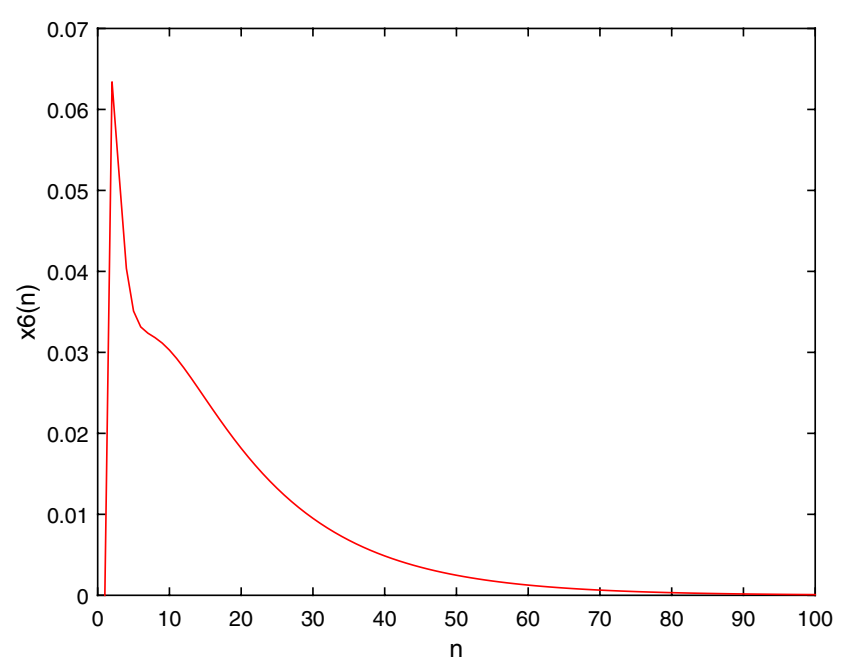

(f)

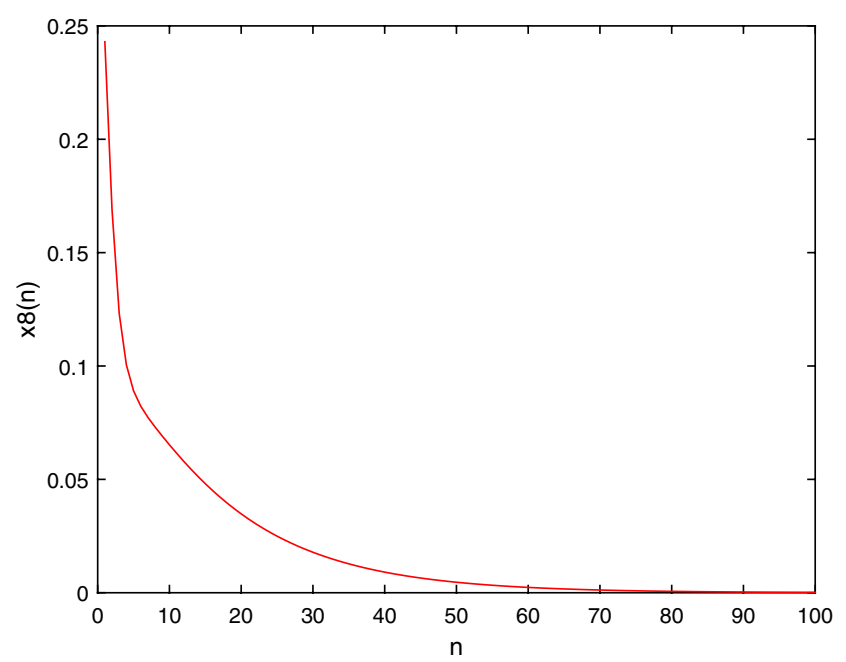

(h)

Fig. 4. (Continued) 


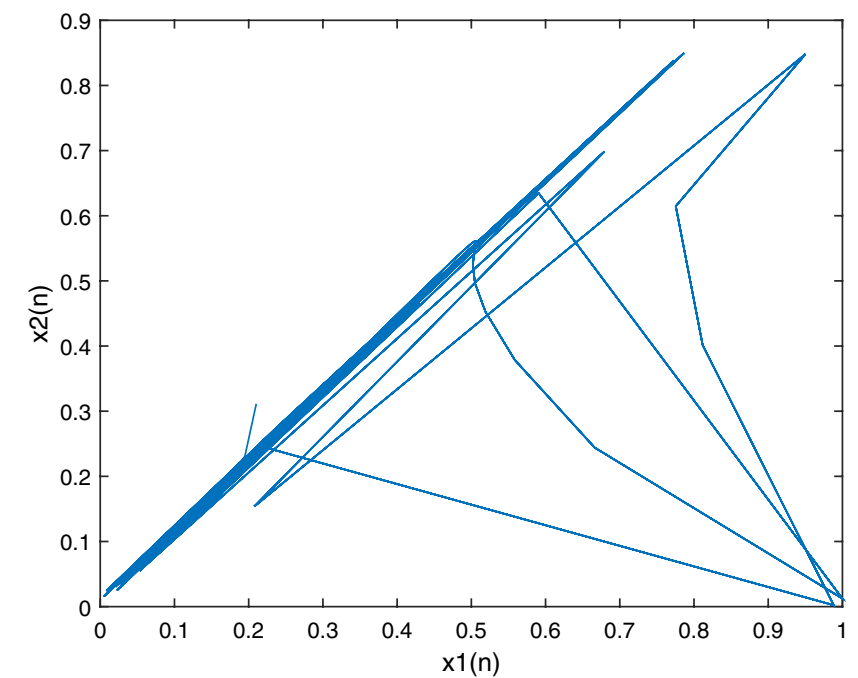

(a)

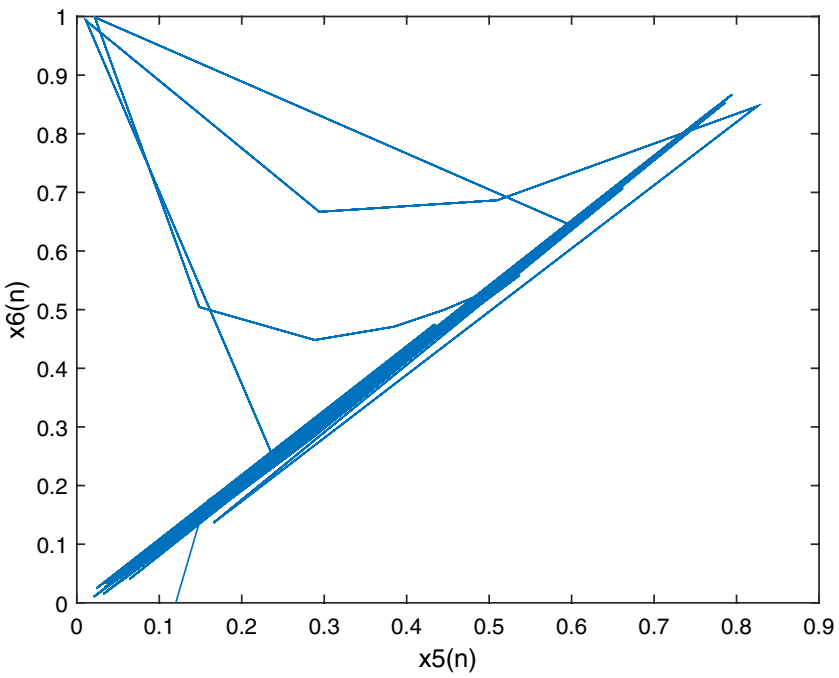

(c)

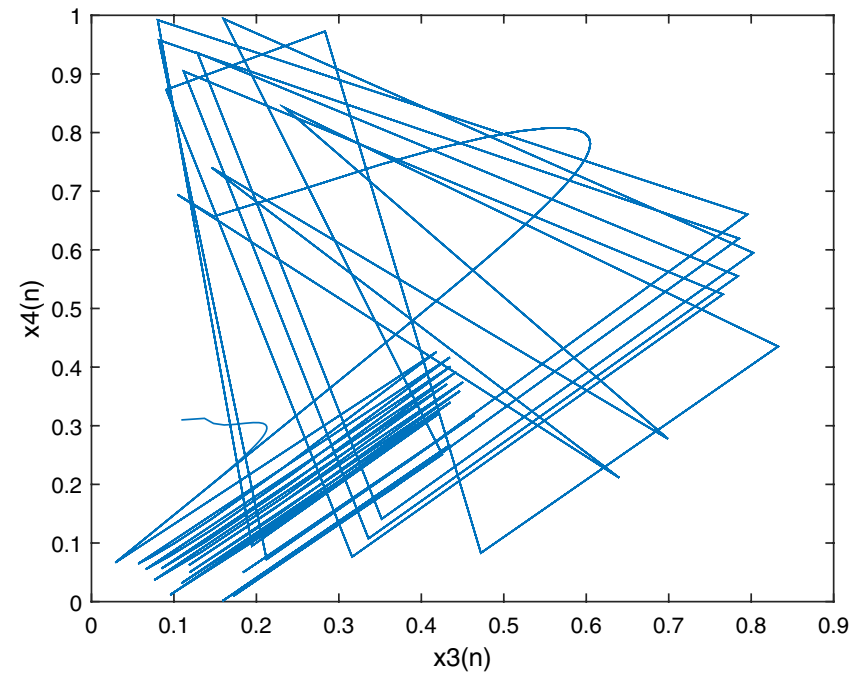

(b)

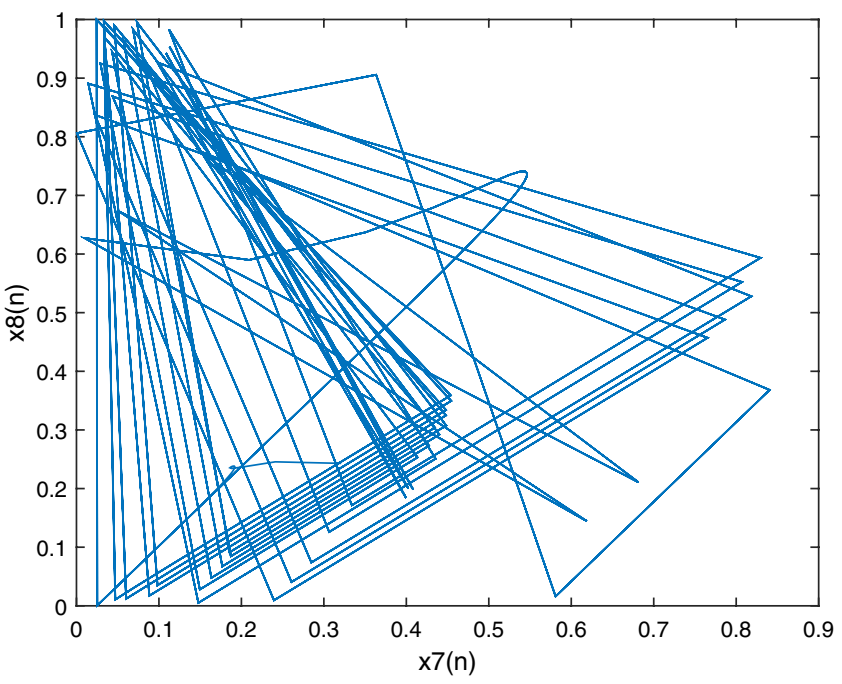

(d)

Fig. 5. The phase diagram of periodic attractors, (a) $x_{1}(n)-x_{2}(n)$, (b) $x_{3}(n)-x_{4}(n),(\mathrm{c}) x_{5}(n)-x_{6}(n),(\mathrm{d}) x_{7}(n)-x_{8}(n)$, (e) $x_{1}(n)-x_{2}(n)-x_{3}(n)$, (f) $x_{4}(n)-x_{5}(n)-x_{6}(n)$ and (g) $x_{6}(n)-x_{7}(n)-x_{8}(n)$. 
Constructing Discrete Chaotic Systems with Positive Lyapunov Exponents

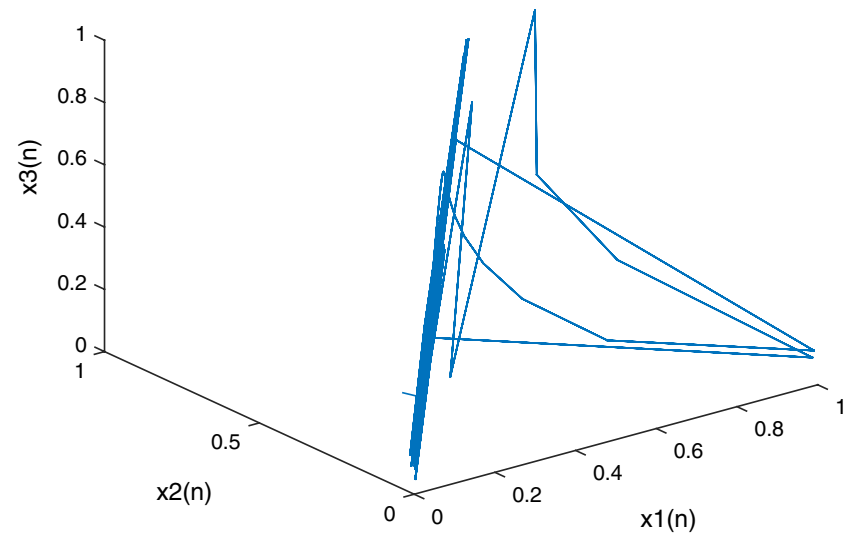

(e)

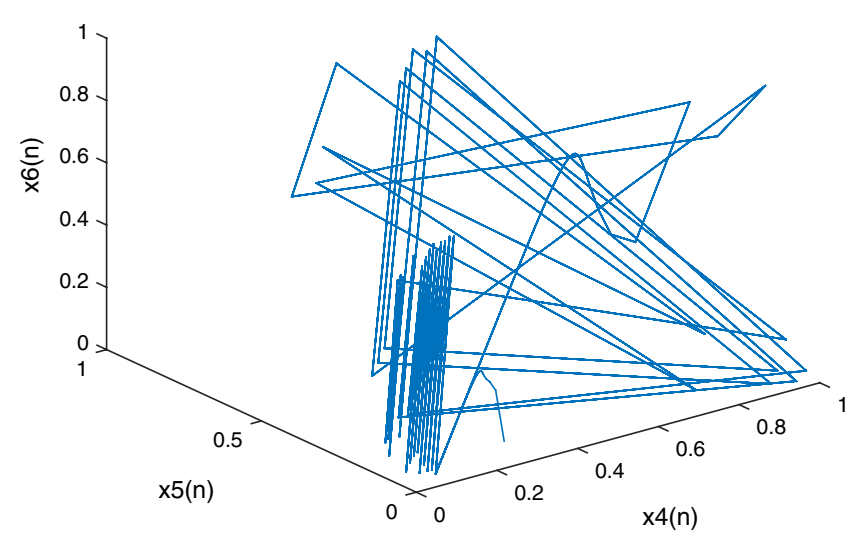

(f)

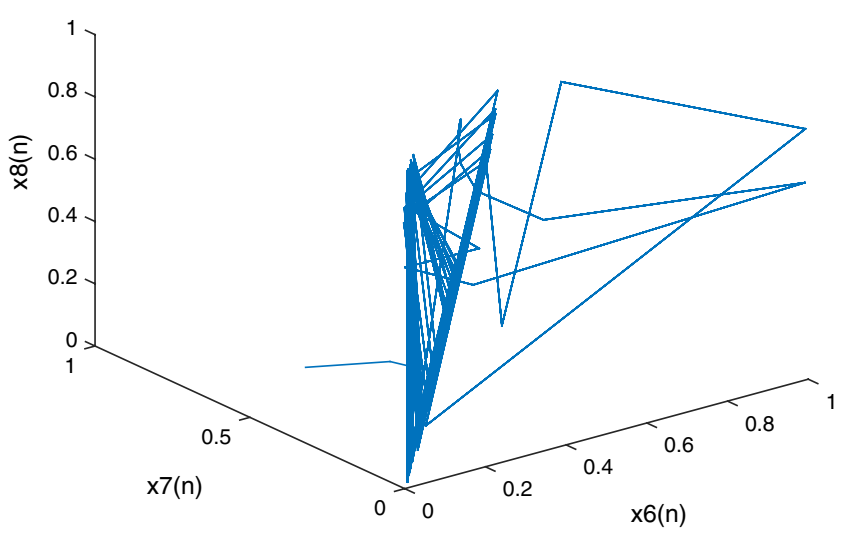

(g)

Fig. 5. (Continued)

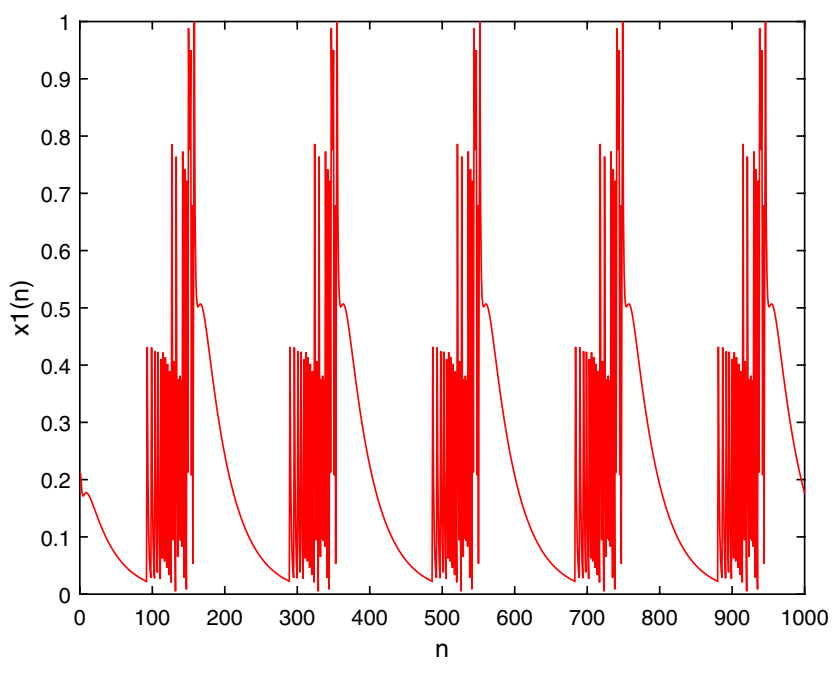

(a)

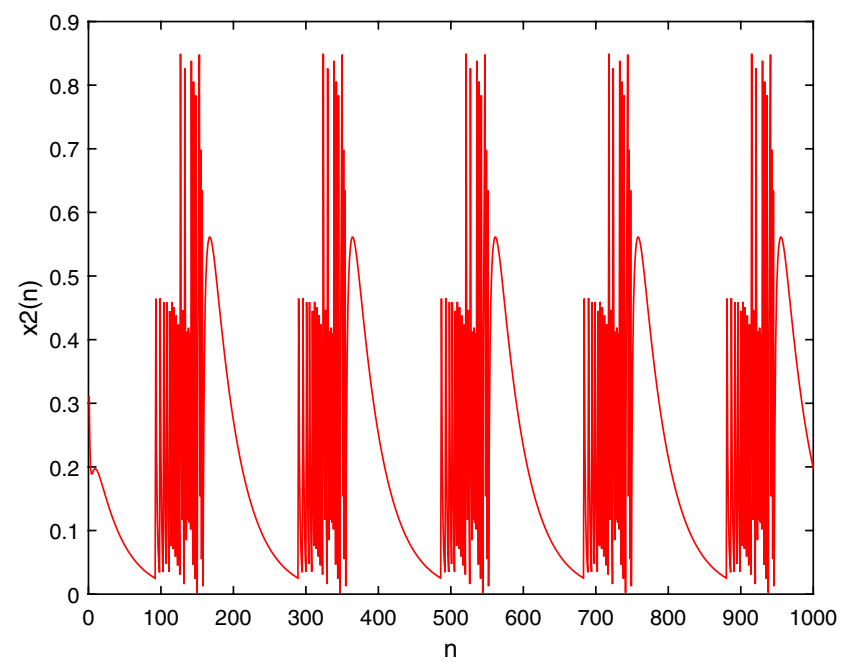

(b)

Fig. 6. The plots of output time series, (a) $x_{1}(n),(\mathrm{b}) x_{2}(n),(\mathrm{c}) x_{3}(n),(\mathrm{d}) x_{4}(n),(\mathrm{e}) x_{5}(n),(\mathrm{f}) x_{6}(n),(\mathrm{g}) x_{7}(n)$ and $(\mathrm{h}) x_{8}(n)$. 
C. Wang et al.

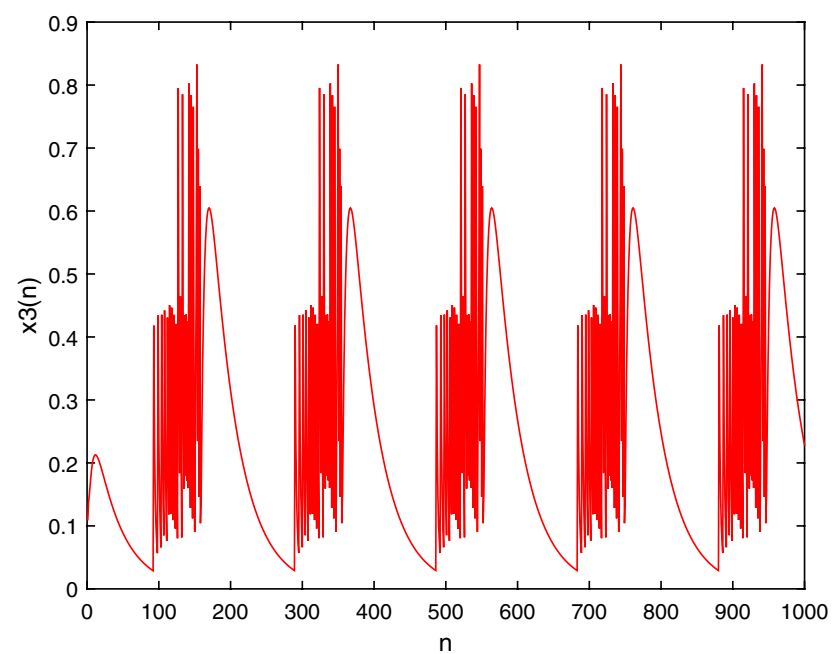

(c)

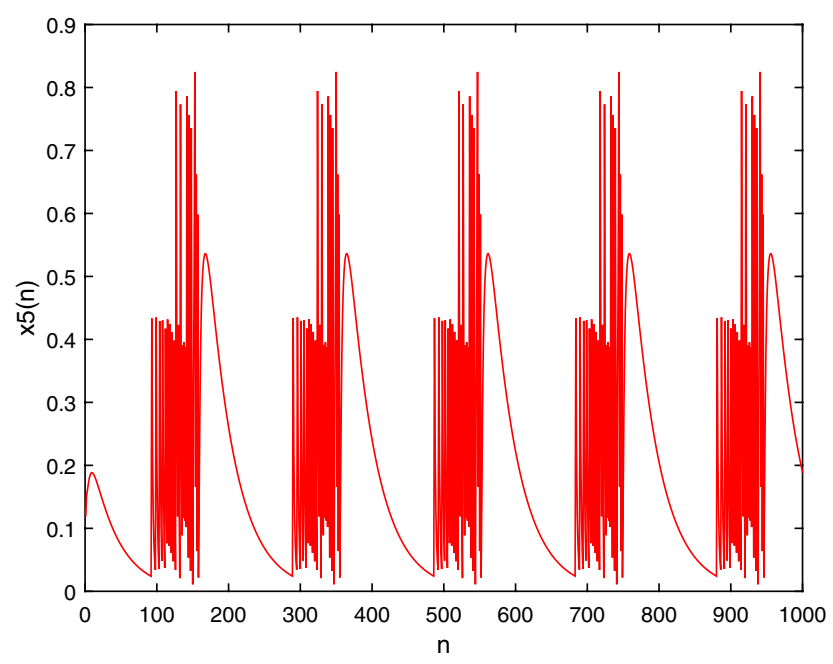

(e)

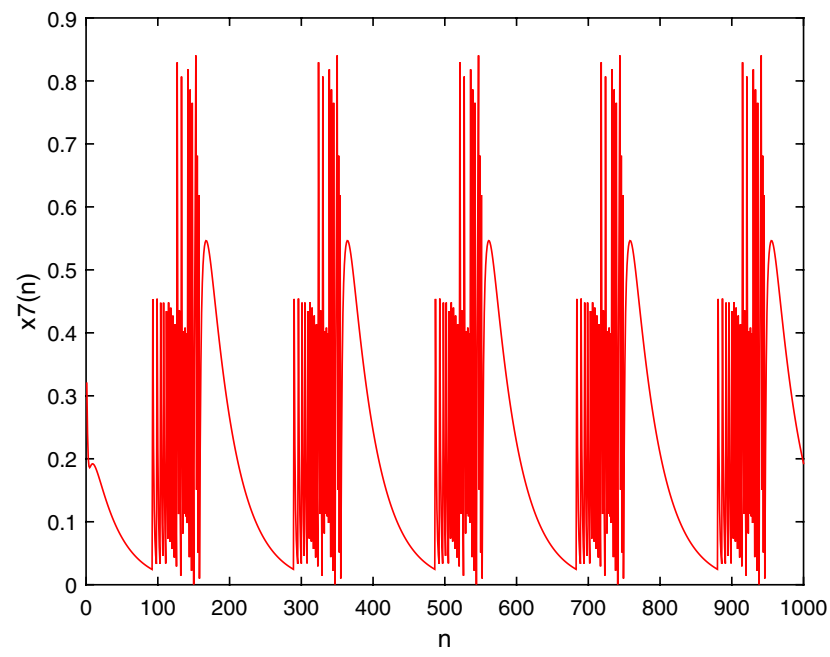

(g)

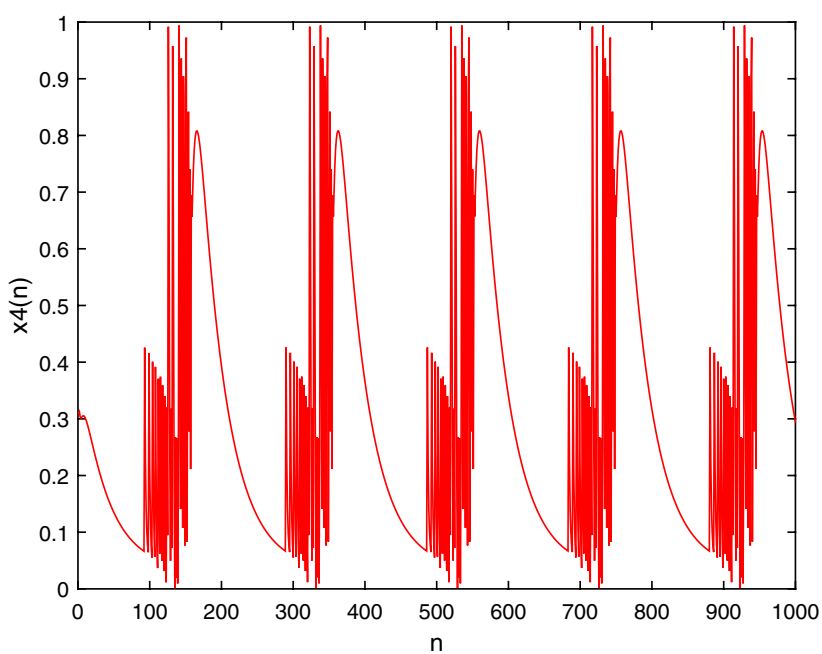

(d)

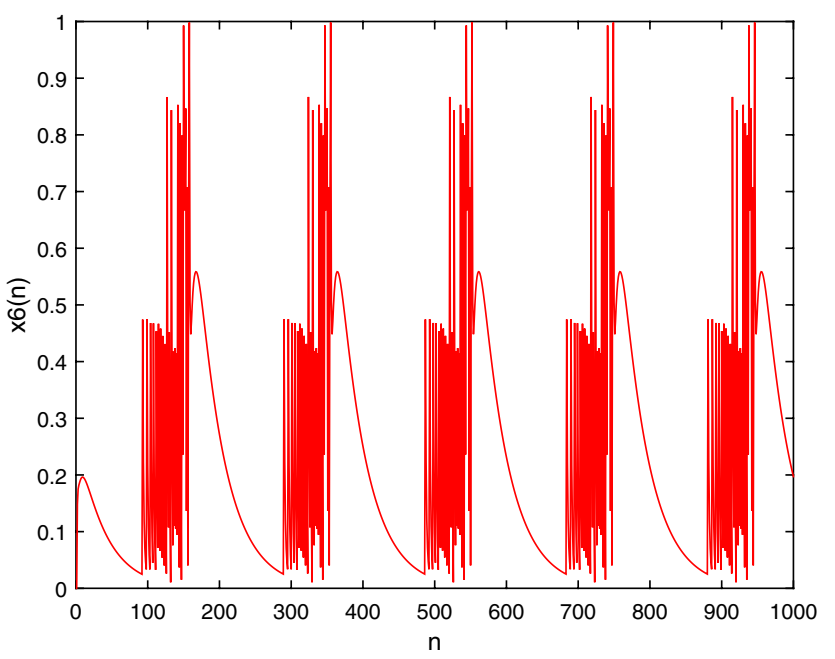

(f)

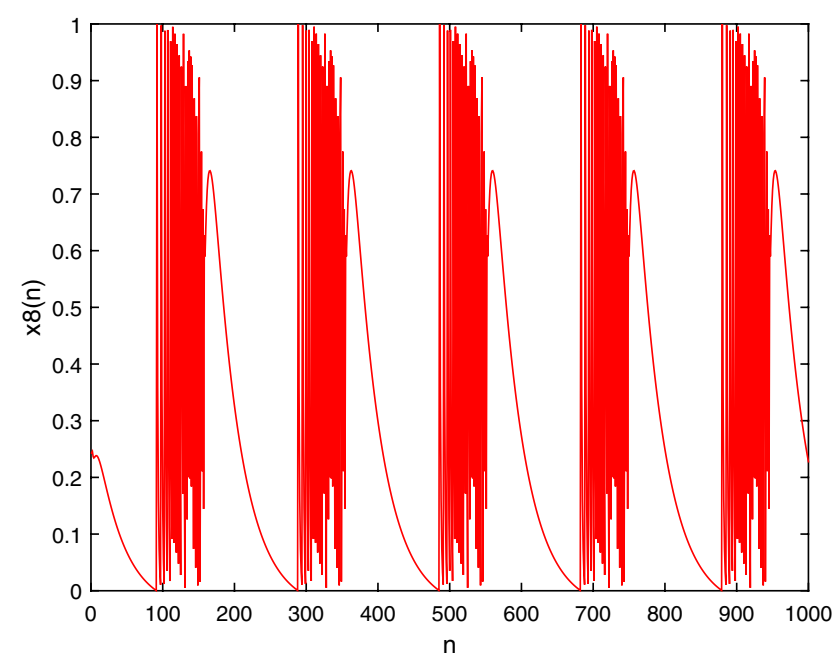

(h)

Fig. 6. (Continued) 
The rounded eight eigenvalues of the matrix $A$ are $0.97,0.83,0.25,0.35,0.42,0.49,0.56,1$, and they are very close to the prearranged values in Step (i). The rounded eight Lyapunov exponents are calculated as $-0.03,-0.19,-1.39,-1.05,-0.87,-0.71$, $-0.58,0$. The phase diagram of the attractors are shown in Fig. 5. The attractors in Fig. 5 are all limit cycles. Although each output sequence is different, they are all periodic sequences. The output time series are shown in Fig. 6 .

\section{Conclusion}

In this paper, a special chaotic discrete system is reconstructed by the given positive Lyapunov exponents. The proposed design method can achieve accurate control of every Lyapunov exponent in the discrete chaotic system since all Lyapunov exponents are preassigned. Different chaotic equations with the same Lyapunov exponents and those with different Lyapunov exponents can be constructed by changing the nonsingular matrix $A$. The proposed method can avoid the nonchaotic state in the multistability, and meet the needs of different chaotic systems in chaotic cryptosystem and chaotic secure communication fields.

\section{Acknowledgments}

This work was supported financially by Natural Science Foundation of China (No. 61471158) and the Innovative Team of the Heilongjiang Province (No. 2012TD007).

\section{References}

Barboza, R. [2007] "Dynamics of a hyperchaotic Lorenz system," Int. J. Bifurcation and Chaos 17, 4285-4294.

Bhatnagar, G. \& Wu, Q. [2012] "Chaos-based security solution for fingerprint data during communication and transmission," IEEE. T. Instrum. Meas. 61, 876887.

Cafagna, D. \& Grassi, G. [2003] "Hyperchaotic coupled Chua circuits: An approach for generating new nmscroll attractors," Int. J. Bifurcation and Chaos 13, 2537-2550.

Chen, G. \& Lai, D. [1996] "Feedback control of Lyapunov exponents for discrete-time dynamical systems," Int. J. Bifurcation and Chaos 6, 1341-1349.

Chen, G., Mao, Y. \& Chui, C. [2004] "A symmetric image encryption scheme based on 3D chaotic cat maps," Chaos Solit. Fract. 21, 749-761.

Chen, A., Lu J., Lü, J. \& Yu, S. [2006] "Generating hyperchaotic Lü attractor via state feedback control," Physica A 364, 103-110.
Ding, Q. \& Wang, J. [2011] "Design of frequencymodulated correlation delay shift keying chaotic communication system," IET. Commun. 5, 901-905.

Habutsu, T., Nishio, Y., Sasase, I. \& Mori, S. [1991] "A secret key cryptosystem by iterating a chaotic map," Workshop on Advances in Cryptology - Eurocrypt. 547, 127-140.

Hu, G. [2009] "Generating hyperchaotic attractors with three positive Lyapunov exponents via state feedback control," Int. J. Bifurcation and Chaos 19, 651-660.

Kapitaniak, T., Chua, L. \& Zhong, G. [1994] "Experimental hyperchaos in coupled Chua's circuits," IEEE Trans. Circuits Syst.-I: Fund. Th. Appl. 41, 499-503.

Kwok, H. \& Tang, W. [2007] "A fast image encryption system based on chaotic maps with finite precision representation," Chaos Solit. Fract. 32, 1518-1529.

Li, Y., Chen, G. \& Tang, W. [2005] "Controlling a unified chaotic system to hyperchaotic," IEEE. Trans. Circuits-II 52, 204-207.

Li, Y., Tang, W. \& Chen, G. [2010] "Hyperchaos evolved from the generalized Lorenz equation," Int. J. Circ. Theor. App. 33, 235-251.

Lin, Z., Yu, S., Lü, J., Cai, S. \& Chen, G. [2015] "Design and ARM-embedded implementation of a chaotic map-based real-time secure video communication system," IEEE. Trans. Circ. Syst. Vid. 25, 12031216.

Pan, J., Ding, Q. \& Du, B. [2012] "A new improved scheme of chaotic masking secure communication based on Lorenz system," Int. J. Bifurcation and Chaos 22, 505-147.

Rech, P. \& Albuquerque, H. [2009] "A hyperchaotic Chua system," Int. J. Bifurcation and Chaos 11, 3823-3828.

Roger, A. H. \& Charles, R. J. [1985] Matrix Analysis, 1st edition (Cambridge University Press, USA).

Rössler, O. E. [1979] "An equation for hyperchaos," Phys. Lett. A 71, 155-157.

Solak, E. [2004] "Partial identification of Lorenz system and its application to key space reduction of chaotic cryptosystems," IEEE. Trans. Circuits-II 51, 557560.

Wang, X. \& Wang, M. [2008] "A hyperchaos generated from Lorenz system," Physica A 387, 3751-3758.

Wang, J., Chen, Z., Chen, G. \& Yuan, Z. [2008] "A novel hyperchaotic system and its complex dynamics," Int. J. Bifurcation and Chaos 18, 3309-3324.

Xie, E., Li, C., Yu, S. \& Lü, J. [2017] "On the cryptanalysis of Fridrich's chaotic image encryption scheme," Signal. Process. 132, 150-154.

Zhu, Z., Zhang, W., Wong, K. \& Yu, H. [2011] "A chaosbased symmetric image encryption scheme using a bitlevel permutation," Inform. Sci. 181, 1171-1186. 